\title{
Tailoring Mission Effectiveness and Efficiency of a Ground Vehicle Using Exergy-Based Model Predictive Control (MPC)
}

\author{
Robert Jane ${ }^{1,2} \mathbb{D}$, Tae Young Kim ${ }^{2}$, Emily Glass ${ }^{2}$, Emilee Mossman ${ }^{2}$ and Corey James ${ }^{2, *(D)}$ \\ 1 Combat Capabilities Development Command (CCDC), U.S. Army Research Laboratory (ARL), \\ Adelphi, MD 20783, USA; robert.s.jane2.civ@army.mil \\ 2 Department of Chemistry and Life Science, United States Military Academy (USMA), \\ West Point, NY 10996, USA; taeyoung.kim@westpoint.edu (T.Y.K.); emily.glass@westpoint.edu (E.G.); \\ emilee.mossman@westpoint.edu (E.M.) \\ * Correspondence: corey.james@westpoint.edu
}

check for updates

Citation: Jane, R.; Kim, T.Y.; Glass, E.; Mossman, E.; James, C. Tailoring Mission Effectiveness and Efficiency of a Ground Vehicle Using Exergy-Based Model Predictive Control (MPC). Energies 2021, 14, 6049. https://doi.org/10.3390/en14196049

Academic Editor: Xiaolin Wang

Received: 19 August 2021

Accepted: 14 September 2021

Published: 23 September 2021

Publisher's Note: MDPI stays neutral with regard to jurisdictional claims in published maps and institutional affiliations.

Copyright: (c) 2021 by the authors. Licensee MDPI, Basel, Switzerland. This article is an open access article distributed under the terms and conditions of the Creative Commons Attribution (CC BY) license (https:/ / creativecommons.org/licenses/by/ $4.0 /)$.

\begin{abstract}
To ensure dominance over a multi-domain battlespace, energy and power utilization must be accurately characterized for the dissimilar operational conditions. Using MATLAB/Simulink in combination with multiple neural networks, we created a methodology which was simulated the energy dynamics of a ground vehicle in parallel to running predictive neural network (NN) based predictive algorithms to address two separate research questions: (1) can energy and exergy flow characterization be developed at a future point in time, and (2) can we use the predictive algorithms to extend the energy and exergy flow characterization and derive operational intelligence, used to inform our control based algorithms or provide optimized recommendations to a battlefield commander in real-time. Using our predictive algorithms we confirmed that the future energy and exergy flow characterizations could be generated using the NNs, which was validated through simulation using two separately created datasets, one for training and one for testing. We then used the NNs to implement a model predictive control (MPC) framework to flexibly operate the vehicles thermal coolant loop (TCL), subject to exergy destruction. In this way we could tailor the performance of the vehicle to accommodate a more mission effective solution or a less energy intensive solution. The MPC resulted in a more effective solution when compared to six other simulated conditions, which consumed less exergy than two of the six cases. Our results indicate that we can derive operational intelligence from the predictive algorithms and use it to inform a model predictive control (MPC) framework to reduce wasted energy and exergy destruction subject to the variable operating conditions.
\end{abstract}

Keywords: exergy; model predictive control (MPC); neural network (NN); multi-domain operations (MDO)

\section{Introduction}

Battlefield systems, whether they are vehicles, personnel with equipment, shelters, weapon systems, or a combination, all require substantial amounts of energy to ensure success. Because energy supplies are finite and must be constantly re-supplied, managing and promoting efficiency will serve to increase resiliency, survivability, flexibility, and longevity of combat forces. Mission effectiveness continues to be the primary consideration for deciding how, where, and when the Army will allocate their available resources in support of current and future military operations. Conversely, efficiency considerations are typically a secondary consideration when designing combat systems and planning operations. With so much overlap between these opposing forces, effectiveness and efficiency, there is opportunity to design solutions that ensure both objectives are accomplished simultaneously.

Figure 1 depicts two M1-A2 Abrams tanks being transported by a C5 Galaxy which can carry a maximum of 140 tons. Each Abrams weighs approximately 67.6 tons; the C5 
is approaching its maximum gross weight capacity by transporting the two tanks. Transporting the tanks using this method is an inefficient waste of resources if you are energy constrained. If constrained by time, however, this is the most effective use of the available resources such that the Army can quickly get systems into a combat theater. Efficiency and mission effectiveness represent trade-offs. One can typically achieve mission effectiveness or efficiency, but it is difficult to achieve both concurrently. Mission effectiveness and efficiency, from a military perspective, is a multivariate challenge driven by time, space, the environment, and other operational considerations which require new and advanced methods to evaluate and optimize the available energy usage in real-time.

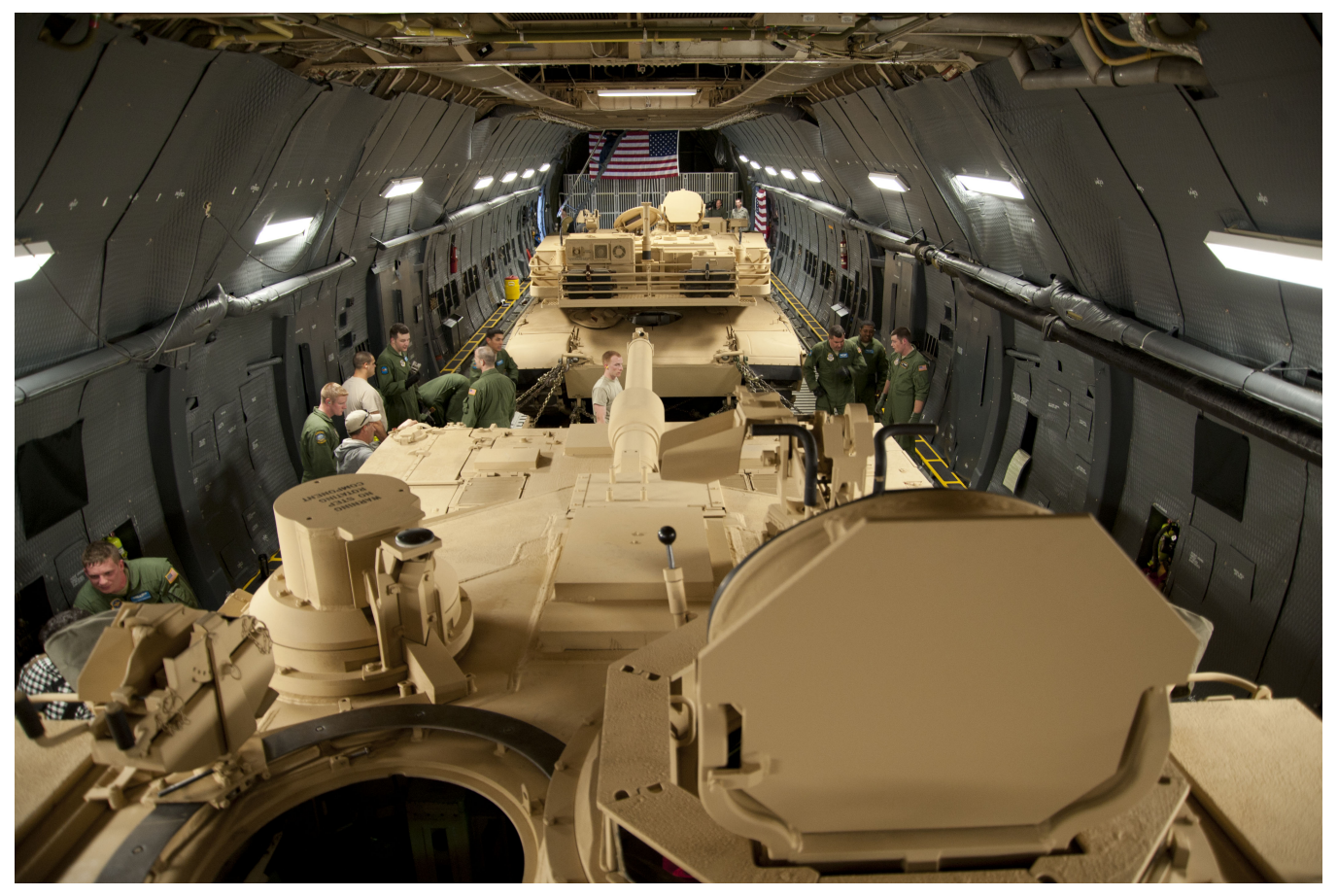

Figure 1. Two M-1 Abrams tanks from Aberdeen Proving Ground, Md., are loaded in the cargo area of a C-5M Super Galaxy assigned to Dover Air Force Base, Del. (U.S. Air Force photo/Lt. Col. Chad E. Gibson) [1].

To achieve this, we envision using open-sourced cloud computing resources, more efficient power electronics with reduced cost and increased efficiency, reduced order modeling, and simulation practices which accurately characterize the dissimilar energy flows. Ultimately, we exploit Artificial Intelligence (AI) and Machine Learning (ML) to create and deploy lower-level control and optimization algorithms for a "box" to be used to inform more energy optimal decisions, supporting maximum mission effectiveness and efficiency. Using MATLAB/Simulink in combination with various Neural Networks (NN) we developed algorithms capable of (1) predicting the future energy state of the vehicle subject to future mission parameters, and (2) providing recommendations based on the energy/exergy flow characterizations for time series data. A few recent studies in which AI/ML have been successfully deployed to time series predictions include Li et al. [2], Serale et al. [3], Sharma and Kushvaha [4] and Kushvaha et al. [5]. Such technical work have shown that accurate time series predictions can be made to improve design and control of dissimilar systems. The recommendations could be provided to the battlefield commanders to make more energy optimal decisions, or used to inform the individual control loops and adaptability to alter the performance of a vehicle. We implemented a Model Predictive Control (MPC) based algorithm which was informed by the NNs to more optimally actuate a Thermal Management System (TMS) to better accommodate the variable mission parameters. The remainder of this section will provide a brief review of 
literature concerning prediction-based algorithms, followed by energy and exergy flow optimization and control.

\subsection{Prediction-Based Algorithms}

To get a full understanding of the dissimilar energy flows within each asset, the copious amount of real-time data must be analyzed and characterized. Additionally, a thorough understanding of the diverse set of operating conditions and mission objectives must be known and understood. Military operations are not typically conducted in well-defined areas with useful infrastructure. Communication is not guaranteed, GPS may be limited, and environmental impacts may be ambiguous. Further, the applicable data sets which describe the operation of an asset are likely sparse and non-diverse and subsequently not extensible to vastly different operating conditions. This situation requires sensing key parameters and estimating others in real-time, while training and learning constantly subject to the lack of communication or GPS information for extended and continued operation of the military assets. This ultimately means that we need a way to develop and deploy computationally efficient reduced order mathematical models of our system which accurately portray the energy flow characterizations subject to time, space, the environment or other operational considerations which impact efficiency and mission effectiveness. Thanks to recent advancements in mathematics and computational sciences, AI, ML, and NN based-algorithms are more robust and applicable for developing predictive algorithms aimed at characterizing the dissimilar energy flows throughout an energy network.

An Artificial Neural Network (ANN) [6], more commonly referred to as a NN, is a computational network created to mimic the behavior of the human brain. Input features are passed to individual neurons contained within the input, output, or hidden layers. Weights and biases are assigned to each neuron, and iteratively optimized throughout the training process, the goal of the training process is to identify key behaviors within the input features that correspond to the output or target features of interest. The weights and biases are set accordingly so that the dominate behaviors which likely lead to the target features of interest become prevalent throughout the various NN layers, enabling the NN to mimic the target features.

There are two major types of NNs-shallow NNs and deep NNs. Originally, the term "deep" was first used to describe Residual Neural Networks(ResNets) with fifty or more hidden layers which included skip connections, i.e., alternative paths that skip over other layers within the neural network to avoid vanishing gradients. The term "shallow" generally means the NN has a single hidden layer, while the term "deep" means the NN has two or more hidden layers. Shallow or deep NNs can be used to fit various functions. However, depending on the complexity of the function, a shallow NN will likely require many neurons within its hidden layers which in-turn drastically increases the number of parameters required to define the NN. Conversely, using a deep NN with more than two hidden layers, where the number of neurons may be able to be reduced significantly, leads to a decrease in the number of parameters required to define the NN. There are other differences as well. Deep NNs typically require larger input data sets to fully describe a particular function whereas a shallow NN can get by with less. A shallow NN is more likely to be less extensible than a deep NN for the same reason. Haykin et al.'s [7] book on NNs provided a comprehensive foundation level understanding of the concepts and functions needed to understand and create these types of predictive algorithms.

There are many different $\mathrm{NN}$ variants, each variant was created in response to a particular objective, constraint, or desired application area. The two most prevalent variations of a NN results from (1) the methodology used for training/learning, and (2) selected architecture. In terms of a NN, learning or training is the process in which the weights and biases within the individual layers are computed such that the target feature may be predicted based on the dominate input features. As stated in Haykin et al. [7], there are seven separate learning processes including (1) Error-Correction Learning, (2) Hebbian Learning, (3) Competitive Learning, (4) Boltzmann Learning, (5) Supervised Learning, 
(6) Reinforcement Learning, and (7) Unsupervised Learning. Each learning technique has its advantages and disadvantages, however the net result is a methodology used to determine the unknown weights of basis of the NN.

The second most prevalent variations of NN results from the selected architecture. The architecture defines the default connection pathways between individual layers and neurons of a network. Some commonly used architectures of NN include ResNets, Long Short-Term Memory (LSTM), Radial Basis Functions (RBFs), Recurrent Neural Networks (RNNs), Convolution Neural Networks (CNNs), MultiLayer Perceptron (MLP), and sequence-to-sequence networks [8]. In regards to vehicle based predictive capabilities, neural networks have been applied to a wide range of problems spanning from vehicle state (speed, roll, slip, etc.) predictions to image classification, route planning, and anomaly detection for manned and unmanned ground and aerial vehicles. The subsequent technical work cited will highlight a small subset of the diverse operational range of the various neural network prediction based variations.

ResNets are commonly used for image classification. Jung et al. [9] used ResNets for vehicle classification and localization observations in traffic surveillance systems. Deep residual networks or ResNets were used in combination with joint fine-tuning (JF) algorithms combined with a Drop Convolution Neural Network (DropCNN) and compared to other common image classification networks: VGG16 [10], AlexNet [11] and ResNet50 [12]. Using the proposed method, the models accuracy for classification and localization based tasks were improved when compared to standard ResNets. The classification method proposed outperformed AlexNet and VGG16 and other common ResNet variants. Similarly the localization method also outperformed similar state-of-the-art detectors. Zhang [13] developed ResNets for autonomous vehicle trajectory prediction. The ResNets were used to forecast the vehicle trajectories, which could capture external feature dimensions better than more standard image capture technologies. The raw input picture was then combined with the ResNet trajectories, experimental results indicated that the proposed method outperformed other common residual network architectures including VGG16 and VGG19.

Long Short Term Memory (LSTM) networks are commonly used for timeseries predictions, speech recognition, and in general a process or processes which have sequence dependencies. Yeon et al. [14] developed ego-vehicle speed prediction using LSTMs. Internal vehicle information pertaining to the relative speed, distance to the vehicle ahead (obtained from radar) and relative vehicle location from a GPS and road-way model were used as inputs to construct a LSTM, which was capable of predicting the vehicles speed for the future vehicle states ( $15 \mathrm{~s}$ in the future) from the past $30 \mathrm{~s}$. Using the developed LSTM, the model was evaluated on three separate scenarios including a car following maneuver, a sharp curve, and a full path trajectory collected from real-driving data. The proposed method was compared to a predictive ANN model, constant speed (CS) model, and constant acceleration (CA) model of the vehicle. The results indicated that the proposed method outperformed the CS, CA, and ANN, resulting from lower RMSE values when compared to the experimental data and the output of the LSTMs.

Radial Basis Functions (RBF) are distinguished from other NN variants in that they are more universally deployed and developed to approximate timeseries functions. Chen et al. [15] developed motion planning algorithms for autonomous vehicles using RBFs. The proposed algorithm extracts the perceived operational environment and uses a gradient descent optimization method to train the network to flexibly derive the drivable region for an autonomous vehicle. The method developed was compared to a more standard Rapidlyexploring Random Tree (RRT) method; experimental results indicated the proposed method offered better motion quality and path planning abilities.

Recurrent Neural Networks, or RNNs, are designed to recognize sequential characteristics and patterns to predict future sequences; this could include speech recognition and natural language processing. Liu and Shoji [16] used deep RNNs to predict vehicle mobility. Vehicle mobility was defined as the position of the vehicle at a given point in time and if a vehicle's path was obstructed mobility was reduced. The proposed RNN 
was simulated using a large-scale taxi mobility data set [17]. The developed RNN was compared to multiple Markov-based Models (MMs) and evaluated using the F1 score which is a measure of the ability of an algorithm to be precise and provide recall ability. The F1 score of the proposed RNN was larger than the F1 score for the MMs simulated. Larger F1 scores indicate better precision and recall capabilities, indicating the proposed method leads to better predictive ability regarding vehicle mobility.

Convolution Neural Networks (CNNs) are primarily used for image processing, classification and segmentation of data. Zheng et al. [18] used CNN for performing drive cycle predictions for plug-in hybrid electric vehicles. Drive cycles were first separated into six different types using k-shape clustering and compared to a k-means algorithm which is more traditionally used as the standard method for clustering drive cycles. Using the results of the k-shape clustering, a CNN was developed to predict the different types of drive cycles, input features were extracted without human assistance, i.e., no human experience was used to derive applicable input features. The CNN was subsequently simulated and compared to the more traditional method of k-means. The k-shape clustering based CNN algorithm outperformed the k-means method. Higher prediction accuracy was obtained. The CNN was then applied to an energy management strategy for a Plug-in Hybrid Electric Bus (PHEB); the results again showed that the k-shape clustering based CNN outperformed the traditional method which lead to more optimal energy usage capabilities for the PHEB.

MultiLayer Perceptrons (MLPs) are typically used for implementing supervised learning, whereas input-output pairs learn model dependencies. It is a well-known fact that predictive algorithms are challenging to develop when applied to deterministic events. Yoon et al. [19] developed a MLP for probabilistic lateral motion prediction of surrounding vehicles. The MLP-based solution was developed to model the target lane and trajectory of a vehicle. The MLP generated probabilistic forecasts which depict possible vehicle trajectories in each lane given the current vehicle position and the past vehicle position. Simulated traffic data was used for training. The results indicated that the proposed algorithm was able to detect lane-changes one and half seconds earlier than existing methods and three seconds before lane crossing with 90\% accuracy. This indicated that an MLP could be used to improve vehicle control and safety based algorithms for autonomous vehicle controls.

Regardless of the selected neural network architecture, NNs are powerful "blackbox" predictive based models. NNs generally lack specifics regarding the knowledge of a systems' dynamics and are thus perhaps less extensible than an state observer. This assumes that the training data-set is non-diverse and covers a limited range of the systems operational environment. If this is not the case, then a NN can likely generate comparable predictions similar to an ordinary state observer. A NN may also be retrained while in operation, thus providing improved extensibility, adaptability, and functionality. An observer is a specific mathematical representation of a predictor which uses knowledge of a dynamical system's states to predict future states of a system. For a well defined system, observers can be designed and deployed to improve control and performance of a system, examples include Kalman filter and sliding mode control.

McBride et al. [20] compared the uses of an ANN and observer for estimating vehicle tire-road contact forces. Tire-road contact forces play an important role in performance of the vehicle, without sufficient contact force, a ground vehicle may be unable to achieve mobility. Insufficient contact force in the lateral or longitudinal directions can cause vehicle instability. These types of forces can be measured, however the instrumentation required to measure such forces is expensive, and it is not suitable for a production vehicle. Using observer theory which accounted for the systems nonlinearities and stochastic states, an observer was constructed and compared to an ANN. The results indicated that the observer out preformed the ANN with higher accuracy. In this case the observer is more extensible than the ANN, because the observer states converge toward the plant states as direct result of the observer states containing the plant states. If subjected to different conditions the observer is expected to track the tire-road contact forces. Conversely, the ANN would have degraded capabilities, which could be overcome by training the 
neural network on a more diverse training data-set or by adaptively retraining the ANN in response to degraded performance error. Broad et al. [21] proposed a robust observer based on $H_{\infty}$ and combined it with a NN to estimate vehicle roll angle. The NN was tested alone, and also combined with the observer. The results indicated that the NN could estimate the general trends of vehicle roll angle, however there were notable noise and errors. The NN was then combined with the $H_{\infty}$ observer, vehicle roll angle could be further estimated with reduced noise and errors.

The net goal of deploying any type of predictor is ultimately to mimic the behavior of a process or processes and subsequently derive some type of operational intelligence or knowledge. When the predictive algorithms are then coupled with an optimization routine or a control loop, more advanced, robust, and extensible control algorithms can be developed and deployed, thus increasing mission effectiveness or efficiency. In the case of vehicles, this generally leads to improved capabilities or more stable operation subject to the variable operational conditions.

\subsection{Energy and Exergy Flow Optimization and Control}

Every fielded asset deployed by the military can be abstracted to the point in which it can be considered a self contained energy network, which possesses energy storage, generation, dissipation, and the ability to interconnect to external energy networks, also known as a microgrid. Each asset is fundamentally governed by a set of complex dissimilar engineering disciplines with no uniform language for expressing energy flow. Every asset is subsequently limited by its own energy generation, storage, and dissipation capabilities. As such many of the assets utilize control algorithms which are purely based on the First Law of Thermodynamics (FLT). The FLT states that energy must be conserved as shown in Equation (1),

$$
\Delta E_{s y s}=m \cdot\left(u_{2}-u_{1}\right)+\frac{1}{2} \cdot m\left(v_{2}^{2}-v_{1}^{2}\right)+m \cdot g \cdot\left(z_{2}-z_{1}\right)
$$

where $m$ is mass, $g$ is the acceleration due to gravity, $u$ represents internal energy, $v$ the velocity, $z$ the height relative to some reference, and the subscripts 1 and 2 are used to denote the difference in states of the system. The first term on the right hand side of the equation represents the difference of the internal energy, the second term represents the difference in kinetic energy, and the final term represents the difference in gravitational or potential energy. Although energy is conserved as the first law requires, not all energy is usable. The Second Law of Thermodynamics (SLT) as defined in Silberberg and Duane [22] as shown in Equation (2),

$$
\Delta S_{\text {univ }}=\Delta S_{\text {sys }}+\Delta S_{\text {surr }} \geq 0
$$

where $\Delta S_{\text {univ }}$ is the entropy of the universe, $\Delta S_{s y s}$ is the entropy increase or decrease of a system, and $\Delta S_{\text {surr }}$ is the entropy transfer from the surrounding environment. Keep in mind that if a process is reversible, there isn't a change in total entropy hence the greater than or equal to sign. The SLT establishes entropy, simply, it states that a process occurs in a certain direction and that the movement of energy can be reversible or irreversible. Irreversible energy loss, increasing the entropy of the universe, is the basis for unusable energy in a process. The combination of the FLT and the SLT leads to exergy, or usable work. In thermodynamics theory the term exergy is commonly used to assess the efficiency of a process. As such, exergy is typically used within the design process but not necessarily used for final implantation of control algorithms. The remainder of this section will present technical work featuring energy or exergy based optimization for design and control.

Control systems were developed to reach and maintain desired set-points in processes as early as 2000 years ago [23]. The volume of literature demonstrating methods for improving efficiency in mechanical, chemical, electrical, or combined processes using rigorous optimization and control is expansive and continues to this day. For the sake of brevity here, this manuscript will not focus on the history of this topic in detail. 
Although the concept of exergy, or useful work, is the basis for a large number of system analysis studies focused on improving efficiency, there are relatively fewer studies that focus on exergy as a basis for control and optimization meant to improve efficiency [24]. The work of James et al. provides a detailed history of instances in the literature where exergy was successfully used as a basis for optimization and control. The work accomplished to date in this field, although it is relatively limited in comparison, yields the conclusion that exergy based optimization and control can provide a comparative advantage over similar energy based methods. This study demonstrates that exergy based optimization and control is vital to improving efficiency in military energy consuming/producing networks. Further, the authors are unaware of any study that used neural networks to inform exergy based optimization and control. This work intends to fill that gap in the literature.

\subsection{Overview}

The remainder of this technical document is arranged as follows: Section 2 will present the mathematical models used to construct the individual vehicle components, Section 3 describes the individual predictive algorithms in combination with our Model Predictive Control based strategy, Section 4 describes our selected set of mission parameters, constraints, and objective, Section 5 presents our results subject to the predefined scenario, and Section 6 will conclude the document by presenting our conclusions and future work.

\section{Modeling}

Our selected vehicle architecture included a small autonomous mobile ground vehicle (MGV), loosely based on the Clearpath Robotics [25] Husky ground vehicle. The original architecture of the MGV as presented in Jane et al. [26] was used to develop and deploy predictive algorithms for characterizing the energy/exergy flow for the current state, and future state of the vehicle subject to a variable set of mission parameters. The vehicle architecture was amended within this study to include six additional components. The additional components permitted energy/exergy flow of the vehicle to be altered subject to time, space, the environment or other operational considerations. The previous vehicle architecture lacked this ability as the only room for improvement concerning energy/exergy was directly linked to the controller design. In this case, the six additional components could be actuated differently subject to the mission parameters to elicit a significant operational change, and thus consume more or less energy/exergy.

The selected MGV architecture includes a battery, two separate types of power converters, two DC spinning machines, a mechanical differential, four wheels, two of which were driven, a thermal compartment, a cold plate, coolant pump, chiller, and coolant tank as shown in Figure 2. The cold plate, coolant pump, chiller, and coolant tank make up a thermal management system (TMS), used to regulate the temperature of the vehicle's battery. The newly introduced components will be described in greater detail within the subsequent subsections. The dynamic equations for the battery, power converters, DC spinning machine, differential, wheel, vehicle body, and thermal compartment can be referenced within Jane et al. [26]. 


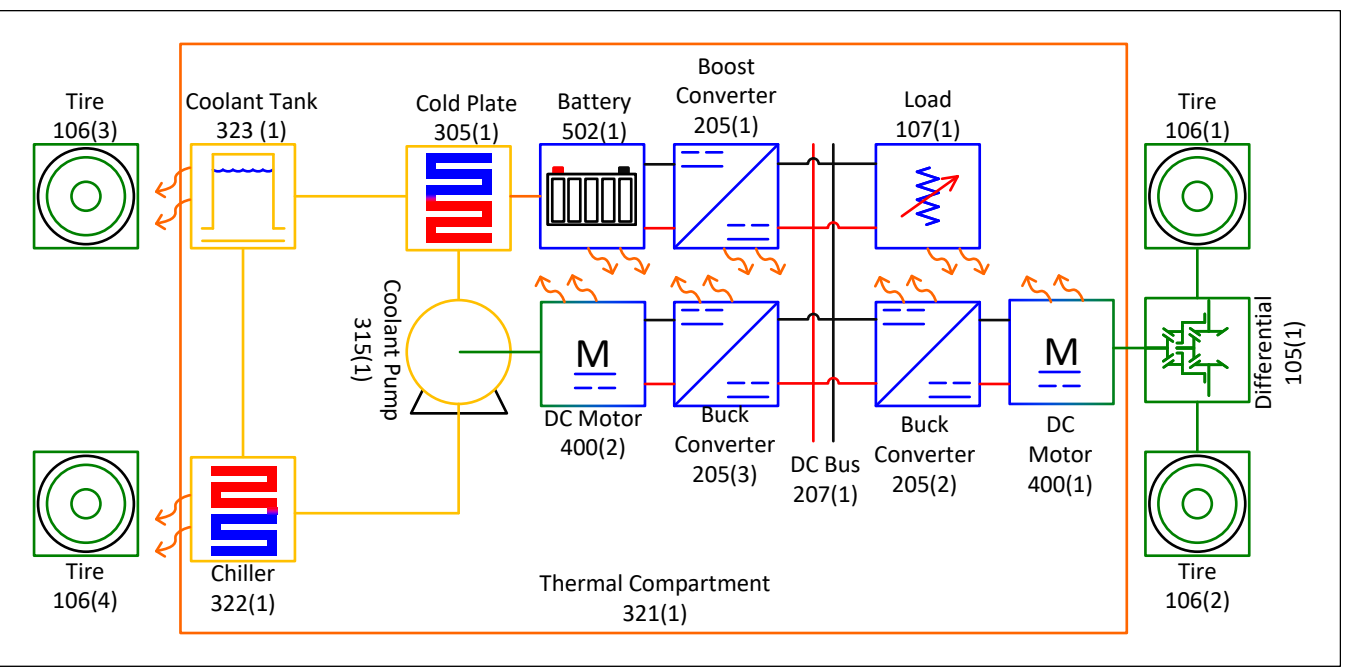

Figure 2. The selected vehicular architecture consists of a battery, three DC/DC power converters, two DC motors, a differential used to drive two separate wheels and a TMS consisting of cold plate, coolant pump, chiller, and coolant tank. Each electrical, electromechanical, or thermal fluid based component has a separate thermal network each of which is contained within the thermal compartment. See Appendix A.1 for explanation of labeling.

\subsection{Cold Plate}

The cold plate was constructed using a combination of Simscape thermal and thermalfluid based blocks. Simscape's Pipe (TL) [27] was used to model a rigid conduit for fluid flow while dynamic compressibility and fluid inertia were ignored. The fluid flow within the component is characterized by an input and output temperature, pressure, and mass flow rate assuming energy was conserved. Equation (3) describes the internal temperature of the thermal fluid,

$$
V \frac{d(\rho \cdot u)}{d t}=\phi_{A}+\phi_{B}+Q_{H}
$$

where $V$ is the volume of liquid within the pipe, $\rho$ is the density of the fluid, $u$ is the internal energy of the working fluid, $\phi_{A}$ and $\phi_{B}$ represent the total energy flow rates through the pipe from port $A$ to port $B$, and $Q_{H}$ is the heat flow rate between the thermal fluid and the pipe's outer wall. $Q_{H}$ can further be broken down into a convective and conductive heat transfer terms, governed by the Nusselt and Reynolds numbers. A separate energy balance is also performed around a solid mass given by Equation (4),

$$
m \cdot C_{p, a v g} \cdot \dot{T}_{i}=Q_{H}+P_{t h, \text { cond }}
$$

where $m$ is the mass of the cold plate, $C_{p, a v g}$ is the average specific heat of the cold plate, $P_{t h, c o n d}$ is the amount of power or heat transferred between the cold plate and the corresponding component in which it is connected to (in this case the battery), and $\dot{T}_{i}$ is the time rate of change of temperature for this particular asset. Heat flow between the thermal fluid and the cold plate is dictated by the gradient of the temperature difference and the fluid flow rate through the pipe. This permits energy to be directly transferred from the MGV's battery to the coolant loop enabling the TMS to regulate the temperature of the battery.

\subsection{Coolant Pump}

The coolant pump was constructed using a Simscape thermal-fluid based block. Simscape's Fixed-Displacement Pump (TL) [28] was used to model a fixed displacement pump controlled by regulating the speed of the DC spinning machine, by controlling the input voltage, regulated by a buck power converter attached directly to the vehicles DC electrical bus. The block has three different variant selections which govern the efficiency of the coolant pump: (1) analytic or tabulated data, (2) input efficiency, or (3) input losses. 
We used the analytic or tabulated data variant in combination with an analytic friction and leakage parameterization to model the coolant pump. The energy balance for the coolant pump is given in Equation (5),

$$
\phi_{a}+\phi_{b}+P_{\text {mech }}=0
$$

where $\phi_{a}$ and $\phi_{b}$ represents the energy flow rates through ports A and B respectively and $P_{\text {mech }}$ is the mechanical power generated as a function of speed and torque, i.e., $P_{\text {mech }}=\tau \cdot \omega$. The hydraulic power is then governed by Equation (6),

$$
P_{\text {hydro }}=\left(p_{a}-p_{b}\right) \frac{\dot{m}}{\rho}
$$

where $p_{a}$ and $p_{b}$ represents the energy flow rates through ports A and B respectively, $\rho$ is the density of the fluid, and $\dot{m}$ is the mass flow rate of the fluid. Equations (5) and (6) assume that pump irreversibility can be neglected (i.e., the ratio between the reversible work and actual work invested on the pump is equal to one), in some cases the pump efficiency may be less than one, in this case an alternative model should be used. Net mass flow rate of the fluid is then given by Equation (7),

$$
\dot{m}=\dot{m}_{\text {ideal }}-\dot{m}_{\text {leak }}
$$

where $\dot{m}$ is the actual flow rate through the coolant pump, $\dot{m}_{\text {ideal }}$ is the ideal mass flow rate subject to the ideal torque, $\tau_{i d e a l}$, and $\dot{m}_{\text {leak }}$ is the leakage mass flow rate subject to the friction torque $\tau_{\text {friction }}$. The ideal mass flow rate and ideal generated torque are then governed by Equation (8) through Equation (9),

$$
\begin{gathered}
\dot{m}_{\text {ideal }}=\rho \cdot D \cdot \omega \\
\tau_{\text {ideal }}=D\left(p_{a}-p_{b}\right)
\end{gathered}
$$

where $D$ is the displacement parameter for the pump, and pressure difference $p_{a}-p_{b}$ assumes port $A$ is the input while port $B$ is the output. The remaining parameters set internal to the Simscape block are then used to analytically define and compute the friction and leakage relationships of the coolant pump.

\subsection{Chiller}

The chiller was constructed using a combination of Simscape thermal and thermalfluid based blocks. Simscape's Pipe (TL) [27] was again used to model a rigid conduit for fluid flow; dynamic compressibility and fluid inertia were also ignored. The fluid flow within the component is characterized by an input and output temperature, pressure, and mass flow rate assuming energy was conserved, thus the same energy balance as provided in Equation (3) remains valid. The chiller was constructed assuming that heat contained within the thermal fluid could be transferred to the ambient conditions subject to Equation (10),

$$
\dot{P}_{t h, r e j}= \begin{cases}\frac{1}{\tau}\left[A \cdot Q(\omega)-B \cdot P_{t h, r e j}\right] & \text { if } \dot{m} \neq 0 \\ 0, & \text { otherwise }\end{cases}
$$

where $\tau$ is a time constant of the chiller, $A$ and $B$ represent scalar coefficients, $P_{t h, r e j}$ is the heat transfer rate from the component to the ambient conditions, and $Q(\omega)$ is the cooling capacity of the chiller governed by Equation (11), i.e., the amount of heat which can be rejected to the ambient conditions defined as a function of the spinning machines angular 
velocity $\omega$, where $Q_{\text {rated }}$ is the rated cooling capacity of the chiller and $\omega_{\text {rated }}$ is the rated speed of the coolant pump.

$$
Q(\omega)= \begin{cases}0, & \text { if } \omega=0 \\ Q_{\text {rated }} \cdot\left[3 \cdot\left(\frac{\omega}{\omega_{\text {rated }}}\right)^{2}-2 \cdot\left(\frac{\omega}{\omega_{\text {rated }}}\right)^{3}\right] & \text { if } 0<\frac{\omega}{\omega_{\text {rated }}} \leq 1 \\ Q_{\text {rated }}, & \text { else } \frac{\omega}{\omega_{\text {rated }}}>1\end{cases}
$$

The chiller then consumes electrical power as a function of Equation (12),

$$
P_{e l c}=\frac{P_{t h, r e j}}{C O P}
$$

where $P_{\text {elc }}$ is an additional parasitic load imposed on the vehicle's DC electrical bus where $C O P$ is the coefficient of performance of the chiller.

\subsection{Coolant Tank}

The coolant tank model was constructed using a Simscape thermal-fluid block. Simscape's Tank (G-TL) [29] was used to model a pressurized tank with variable gas and thermal liquid volume. The complete derivation of the equations that govern the dynamics are provided within MATLAB's documentation. For brevity here, the net result of mass, energy, and momentum balances for the components are provided in Equation (13) through Equation (15),

$$
\begin{gathered}
\dot{p}_{g}\left(\left.\frac{d m}{d p}\right|_{g}+\frac{\rho_{g} V_{l}}{\beta_{l}}\right)+\left(\left.\dot{T}_{g} \frac{d m}{d T}\right|_{g}-\dot{T}_{l} \rho_{g} V_{l} \alpha_{l}\right)=\sum_{i=A_{1}, B_{1}} \dot{m}_{i}+\frac{\rho_{g}}{\rho_{l}} \sum_{i=A_{2}, B_{2}, C_{2}} \dot{m}_{i} \\
\left.\dot{p}_{l} \frac{d U}{d p}\right|_{l}+\left.\dot{T}_{l} \frac{d U}{d T}\right|_{l}=Q_{h_{2}}+\sum_{i=A_{2}, B_{2}, C_{2}} \phi_{i}+\sum_{i=A_{2}, B_{2}, C_{2}} \dot{m} \mathbf{g}(y(i)-y)-h_{l} \sum_{i=A_{2}, B_{2}, C_{2}} \dot{m}_{i} \\
p_{i}+p_{i, d y n}=p_{g}+\rho_{l}\left(y-y_{i}\right) \mathbf{g}
\end{gathered}
$$

where subscripts $l$ and $g$ represent liquid and gas respectively, the term $g$ represents the acceleration due to gravity, $\rho$ for density of the fluid or gas denoted using the appropriate subscripts, $p$ represents the pressure, $T$ for temperature, $y_{i}$ represents inlet/output fluid port heights, $y$ represents the height of the gas-thermal liquid boundary, $\alpha$ represents the isobaric thermal expansion coefficient, $\beta$ represents the isothermal bulk modulus, $A_{1}$ and $B_{1}$ represents the two gas input ports, $A_{2}, B_{2}$, and $C_{2}$ represent one of three possible thermal fluid ports, $\phi$ represents the energy flow rates through the fluid inlets, $Q$ is the heat flow through the thermal port, $h$ is the fluid enthalpy, $U$ is the total energy of the fluid volume, and $p_{i, d y n}$ is the dynamic pressure at each thermal liquid input. The coolant tanks thermal ports are directly connected to a thermal resistance, which impedes heat flow from the pressurized tank to the thermal compartment, assumed to represent that the coolant tank is insulated from the internal components to some degree.

\section{Predictive Algorithms and Control}

Recall that our research has two underlying goals, (1) predicting the future energy use provided forecasted mission parameters, and (2) generating optimized recommendations for improved mission effectiveness and efficiency. Current and future energy use of a vehicle is likely to have sequence dependencies. As such, we sought to develop multiple Long Short Term Memory Networks (LSTMNs) and multiple Shallow Artificial Neural Networks (SANNs). LSTMNs are more computationally intensive, require greater training time and typically require larger and more diverse data sets but achieve greater extensibility or adaptability when compared to a SANN. Using the different NN variants, we assumed we could develop reasonably accurate predictions for the current and future vehicle states, further assuming we also have some observational knowledge regarding the environment 
or other operational considerations. The final research question that we are working to understand is whether we can generate optimized recommendations which improve performance of the vehicle subject to mission effectiveness and efficiency. To achieve this, we implemented a Model Predictive Control (MPC) algorithm which was fed by the individual neural network variants to reduce the exergy destruction rate as a function of the operation of the vehicle and other mission objectives and constraints. More information regarding the SANNs, LSTMNs and MPC will be provided within subsequent sections.

\subsection{Shallow Artificial Neural Networks (SANNs)}

SANNs can produce an accurate description of nonlinear timeseries models in a timely manner with efficient error correction by utilizing one to two hidden layers that add weight and bias to the inputs of the system; these hidden layers, or interposed neurons, provide learnable parameters that effectively train the neural network to output the desired optimized model. In comparison to other NNs, SANNs have lower risks of exploding gradients or vanishing gradients that are often related to training larger neural architectures, thus making them relatively simple to train and use in research applications. These NNs are also much more time efficient when compared to complex networks with more than two nodes that require more extensive training times. Specifically, primarily due to the flexibility of NNs, SANNs have been used to effectively model nonlinear timeseries data similar to the data in this study. Zhou et al. clearly demonstrated the usage of SANNs in a highly nonlinear timeseries system to create a predictive algorithm modeling the groundwater status in millimeters of the Heihi River basin over the span of several decades [30]. In addition to this, SANNs have also been shown to be able to optimize the nonlinear function for Chemical Vapor Infiltration (CVI) parameters of carbon composites in the work of Li Aijun et al. [31]. Thus, SANNs are shown to be optimal to create predictive models and have potential to appropriately characterize the exergy destruction and flow data as a nonlinear timeseries in this study.

\subsection{Long Short Term Memory Networks (LSTMNs)}

Long short-term memory networks are a variations of recurrent neural networks with enhanced capabilities to retain information over multiple time steps. Recurrent neural networks are characteristically defined by their ability to utilize previously generated outputs as input values, along with other new information entered into the network. A node within the network uses a predetermined function or set of functions to perform calculations from input values to generate output values. Output values are continually used with new input values to generate the next output value. Specifically, within long short-term memory networks, each node contains multiple internal states. Within these states, information can be stored for long-term memory and retrieved over many time steps such that information stored in the states, previous outputs, and new inputs are all used in the calculation of the node. Within the node, parameters control how inputs are used by nodes for calculations.

Long short-term neural networks have been utilized and researched in various applications of sequence finding in complex patterns of unknown length. These neural networks have been applied to other highly non-linear systems to identify sequential patterns and interdependent relationships for future predictions of the system through timeseries functions. Fischer and Krauss, through applying long short-term memory networks to predict the financial market, found that the long short-term memory neural networks outperformed the deep neural network, logistic regression analysis, and random forest neural network "with statistically and economically significant returns of 0.46 percent per day, compared to 0.43 percent for the Random Forest (RAF), 0.32 percent for the standard Deep Neural Network (DNN), and 0.26 percent for the logistic regression" [32]. Ahuja et al. found in applying long short-term neural networks to predict domain generated algorithms, this variation of recurrent neural networks outperformed the HMM model, the random forest neural network, and the logistic regression in predicting true positive domain generated 
algorithms [33]. Through this, the retention of state and long-term memory capabilities of long short-term memory networks are highlighted and shown to be effective in sequential learning and generating accurately predictive outputs.

\subsection{Model Predictive Control (MPC)}

Our mathematical models and NNs were constructed in such a way that they were meant to be modular. In order to predict the current state or future state of the vehicle or an individual component, we develop multiple predictive based algorithms to characterize the current and future energy/exergy flows for each dissimilar engineering discipline modeled, chemical, mechanical, electrical, thermal, thermal-fluid, etc, thus the objective function for the MPC algorithms is provided in Equation (16),

$$
J(t)=\sum \dot{\chi}(t)
$$

where $J(t)$ represents the aggregate cost computed as the summation of the exergy destruction rate $\dot{\chi}(t)$ resulting from the operation of each individual component. The individual electrical, electromechanical, and thermal exergetic losses equations are the same as those featured in Jane et al. [34]. We also included the electrical load to operate the chiller as an exergetic loss in that if we did not run the chiller, we would save any electrical energy not used to operate the thermal coolant loop. There are two design variables which are to be optimized subject to the bounds as shown in Equations (17) and (18),

$$
\begin{gathered}
250 \mathrm{rpm} \leq \omega_{d c} \leq 500 \mathrm{rpm} \\
1{ }^{\circ} \mathrm{C} \leq \Delta T \leq 4{ }^{\circ} \mathrm{C}
\end{gathered}
$$

where $\Delta T$ represents the temperature offset and $\omega_{d c}$ represents the controlled speed of the DC spinning machine which drives the coolant pump. The temperature offset was used to generate a temperature error signal which triggers the hysteresis control. The temperature error signal was computed using Equation (19)

$$
T_{\text {err }}=T_{\text {batt }}-\left(T_{a m b}-\Delta T\right)
$$

where $T_{a m b}$ is the ambient temperature, $T_{\text {batt }}$ is the battery's temperature, and $T_{\text {err }}$ is the temperature error. A hysteresis control was then applied to control the temperature of the battery.

The hysteresis was defined such that if $T_{e r r} \geq 1$ the hysteresis or relay will be triggered to cool the battery, the TMS will remain active until $T_{\text {err }} \leq 0.1$, at which point the TMS will be disabled. By changing the temperature offset, the temperature at which the battery was regulated will change. Reducing $\Delta T$ will reduce the time in which the TMS is active, thereby reducing the pure mechanical and electrical exergy losses associated with the duration of time in which the TMS was active. Conversely, if $\Delta T$ was increased the TMS will remain active for longer durations, this will in turn increase the pure exergetic losses resulting from a longer duration of activity. Alternatively, the DC spinning machine's angular velocity $\omega_{d c}$ could increase. Increasing the speed will increase the exergetic losses in magnitude and the duration at which the TMS will remain active will decrease the exergetic losses resulting from a shorter duration of activity. Conversely, decreasing the angular velocity will lead to a reduction in exergetic losses associated with the operating the TMS. However, the TMS is likely to operate for longer durations and exergetic losses could increase.

In any case, the duration and magnitude in which the TMS will be enabled or disabled may increase or decrease as a function of the selected angular velocity of the DC spinning machine or the temperature offset. The exergetic losses of the vehicle will be minimized subject to the event schedule. The event schedule directly corresponds to mission effectiveness or efficiency. The event schedule defined a set of U.S. Army relevant events which may require alteration to the control logic for the TMS to better accommodate mission 
effectiveness. One such event where this is the case is during a reduced ancilliary load operation. During a reduced ancilliary load operation, the vehicle would be operating with a reduced load. Unnecessary actuation of a component or group of components such as the TMS will increase the aggregate electrical load which would reduce mission efficiency. Thus during a reduced ancilliary load operation, the TMS should be controlled such that it is minimally actuated. Using the event schedule in combination with the equations presented within this section the MPC was created and tested. If the predictive algorithms indicated that a particular set of temperature offsets and angular velocity operational conditions resulted in the activation of the TMS, that particular set of operating conditions will be considered infeasible.

To limit the solution space, we broke the simulation epoch into two optimization epochs. First, we will optimize at the beginning of the simulation and then roughly an hour after the start of the simulation we would re-execute the MPC. The first epoch was broken up as shown in Equation (20), while the second was broken up as shown in Equation (21), where

$$
\begin{aligned}
& {\left[\begin{array}{c}
\omega_{d c} \\
\Delta T \\
t
\end{array}\right]=\left[\begin{array}{cccc}
\omega_{d c, 1} & \omega_{d c, 1} & \omega_{d c, 2} & \omega_{d c, 2} \\
\Delta T_{1} & \Delta T_{1} & \Delta T_{2} & \Delta T_{2} \\
0 & 3300 & 3300.5 & 10800
\end{array}\right]} \\
& {\left[\begin{array}{c}
\omega_{d c} \\
\Delta T \\
t
\end{array}\right]=\left[\begin{array}{cccc}
\omega_{d c, 1} & \omega_{d c, 1} & \omega_{d c, 2} & \omega_{d c, 2} \\
\Delta T_{1} & \Delta T_{1} & \Delta T_{2} & \Delta T_{2} \\
3300 & 6900 & 6900.5 & 10800
\end{array}\right]}
\end{aligned}
$$

where $\omega_{d c, 1}$ and $\omega_{d c, 2}$ are the first and second angular frequency setpoints, $\Delta T_{1}$ and $\Delta T_{2}$ are the first and second angular temperature hysteresis variables, and $t$ represents a time vector for which the setpoints will be valid. Using these variables, the MPC routine will then minimize the exergy destruction of the MGV and also accommodate minimal actuation of the thermal coolant loop (TCL) during the reduced ancilliary load event. Our MPC was formulated such that we used a brute force optimization routine in which we predefined each unique possible solution assuming the coolant pump's angular frequency could be controlled to be $\omega_{d c}=250 \mathrm{rpm}, \omega_{d c}=375 \mathrm{rpm}$, or $\omega_{d c}=500 \mathrm{rpm}$, and subjected to $\Delta T=1{ }^{\circ} \mathrm{C}, \Delta T=2{ }^{\circ} \mathrm{C}, \Delta T=3{ }^{\circ} \mathrm{C}$, and $\Delta T=4{ }^{\circ} \mathrm{C}$ temperature hysteresis, i.e., twelve unique solutions for the first and second intervals leading to one-hundred and forty-four unique solutions which may allow the MGV's operation to be optimized for mission effectiveness and efficiency.

\section{Scenario}

A scenario in the context of a microgrid, or more generally an energy network, represents a set of mission parameters, operating conditions, mission loads, and/or drive cycle, all of which impact performance of an asset. In this particular case we choose a small robotic platform which was loosely based on Clearpath Robotics robotic platform for semi-autonomous or autonomous vehicle. We used the same scenario specific information as presented in Jane et al. [26]. For brevity, the individual training and testing data-sets are also provided within this document. In order to develop the different predictive capabilities, we generated two separate data-sets, each data-set contained a mission load profile, a set of operating conditions (temperature, pressure, etc.), road grade, drive cycle, and event schedule. The event profiles and operating conditions were held constant for both the training and testing data-sets, both of which are provided in Figure 3.

In general, each data-set contained a drive cycle which contains two mobility maneuvers, representing a round trip maneuver. In between each mobility maneuver, the vehicle will remain stationary, during which time it will participate in a reduced ancilliary load operation. At this time on-board Government Furnished Equipment (GFE) will be used to conduct some predefined mission. The mission loads are another distinguishing characteristic for each data-set, in addition to separate road grades. 


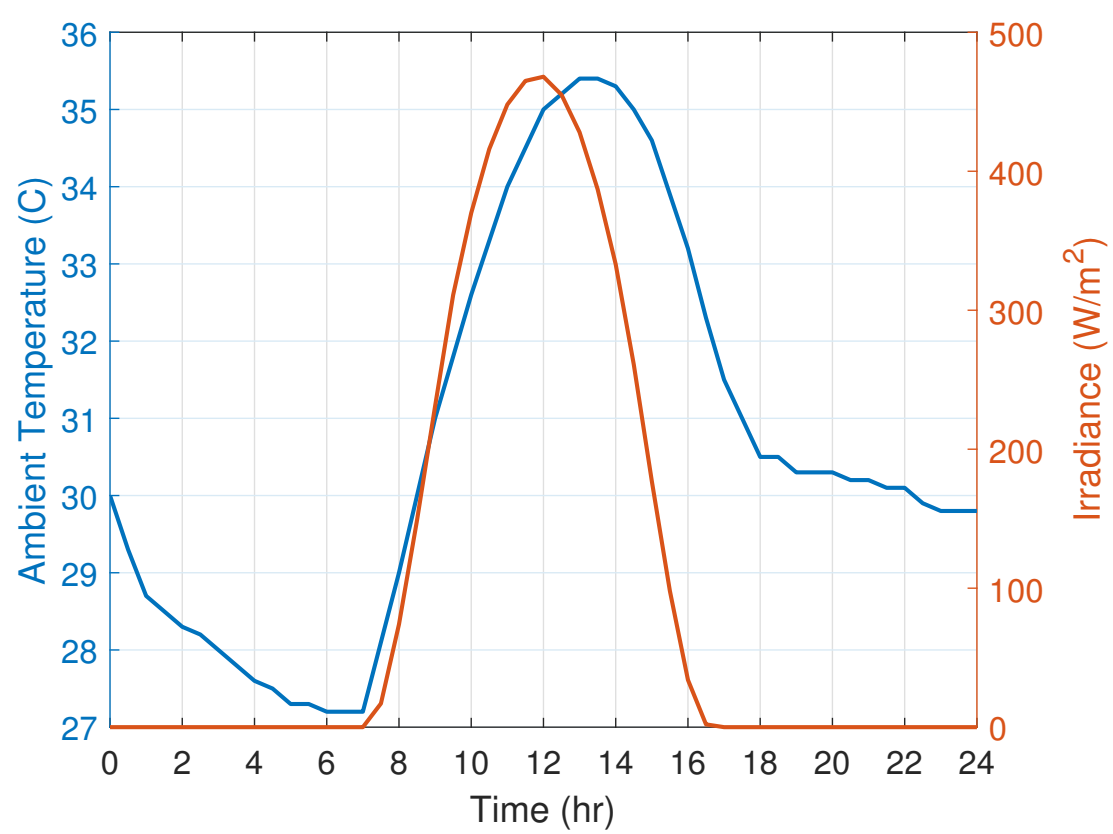

(a) The variable operating conditions consists of a variable temperature and irradiance profile downloaded from NREL's NSRDB tool.

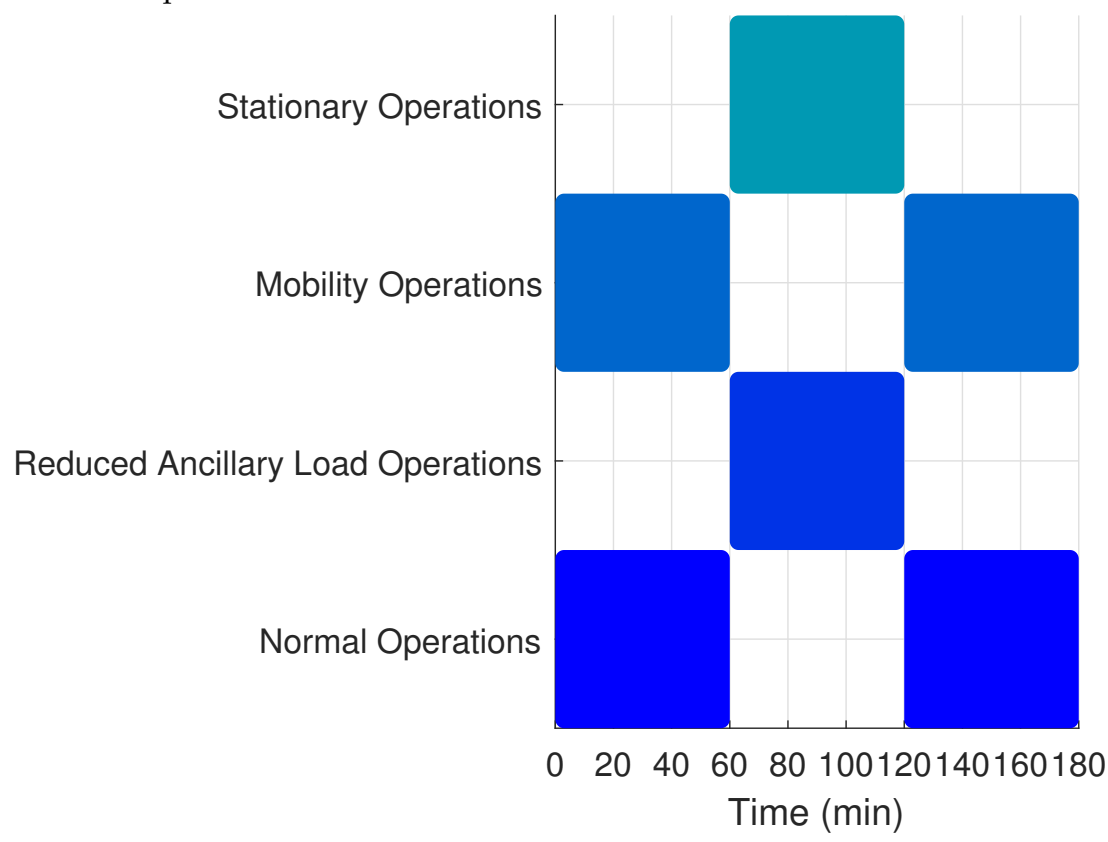

(b) The mission's event schedule. Normal operations is assumed under a mobility maneuver, conversely during a reduced ancilliary load operation, the vehicle should operate with a reduced aggregate electrical load.

Figure 3. A variable set of operating conditions and event schedule which impact performance of the vehicular asset.

The training and testing data-sets are shown in Figure 4 . The training data-set was used for initial development of the individual predictive algorithms. Using the individual drive cycles, road grade, and mission load profiles, the vehicle architecture was simulated. Analysis of each asset's energy and exergy flow were subsequently used to identify pertinent features, used to predict the dissimilar energy and exergy flow features, enabling energy and exergy flow characterization to be developed. The testing data-set was then used for assessing the individual predictive algorithms. Prior to implementing the MPCbased optimization routine described previously, the individual prediction algorithms 
were tested on the testing data-set in order to observe the applicability of the predictive algorithms. If the algorithms performance was poor, MPC based-control would suffer, if acceptable, MPC-based optimization and control could be applied to improve performance of the vehicle.

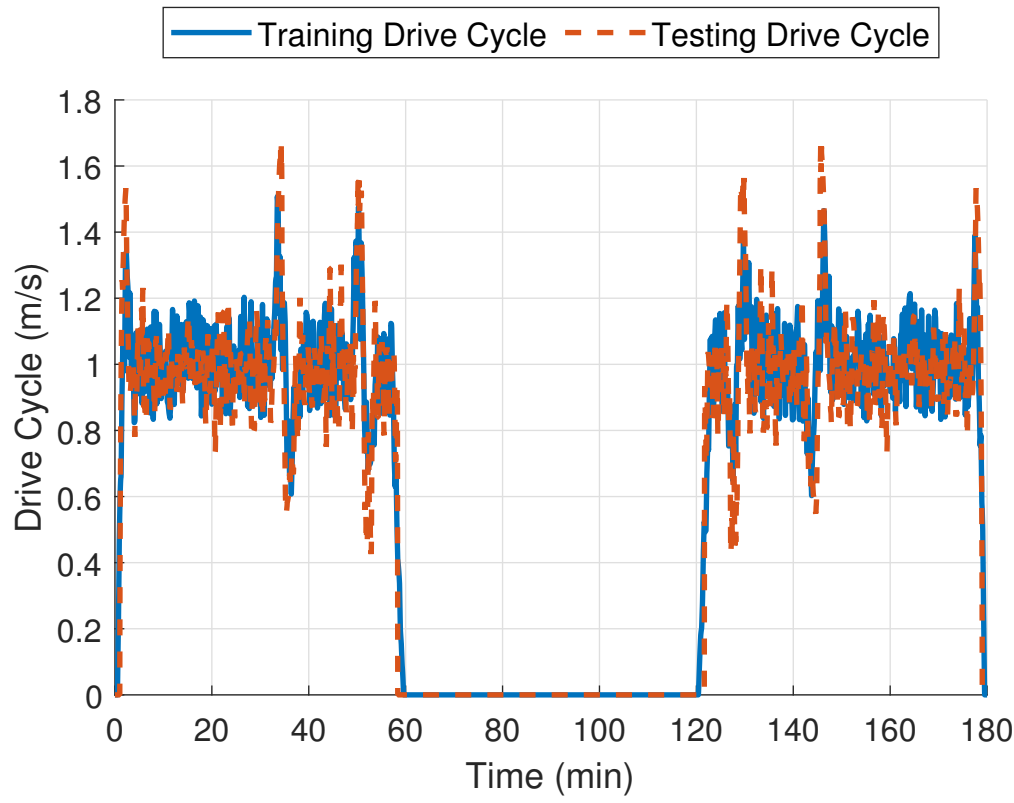

(a) The training and testing drive cycle.

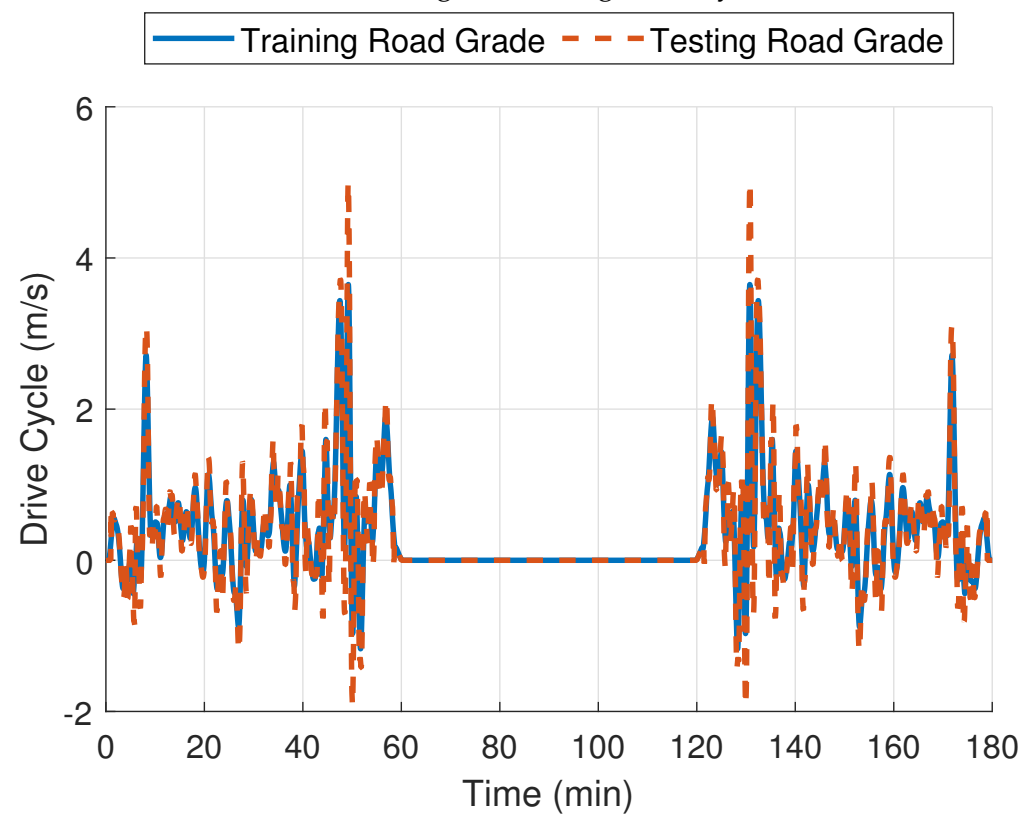

(b) The training and testing road grade.

Figure 4. Cont. 


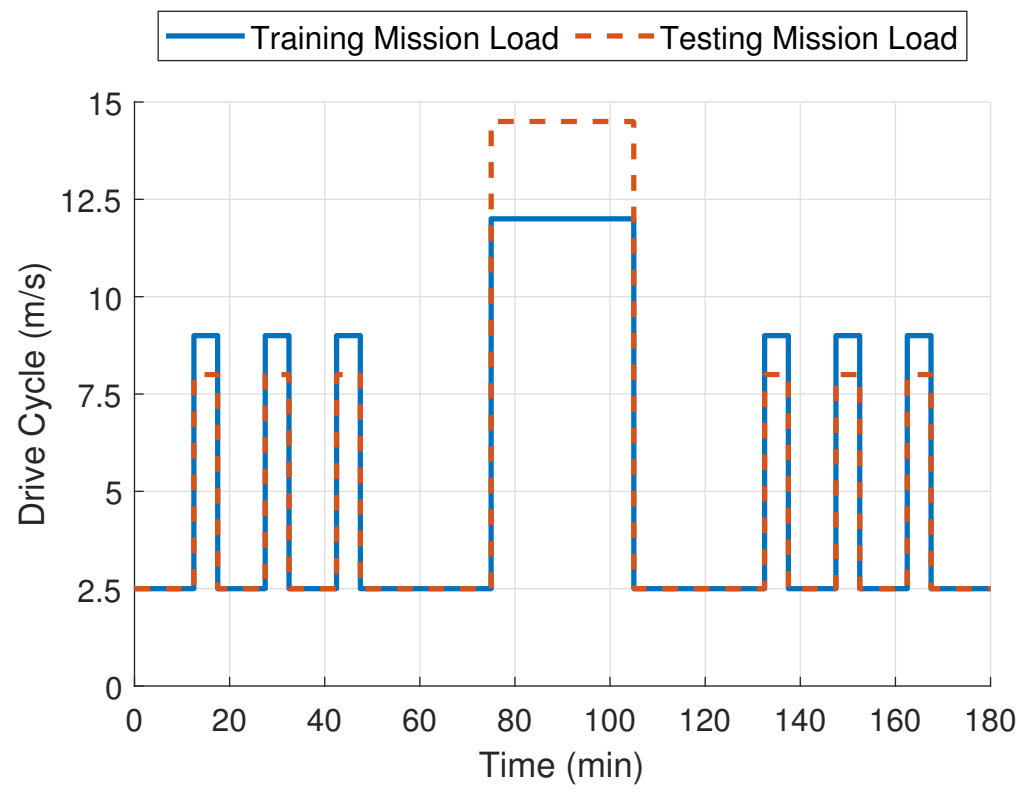

(c) The training and testing mission load current imposed on the MGV's DC electrical bus.

Figure 4 . The training and testing drive cycle, road grade, and mission load profile.

\section{Results}

Prior to testing the predictive algorithms, the MGV was simulated twelve separate times, six times on the training data-set and six times on the testing data-set. The MGV was simulated assuming the TCL would operate at $\omega_{d c}=250 \mathrm{rpm}, \omega_{d c}=375 \mathrm{rpm}$, or $\omega_{d c}=500 \mathrm{rpm}$, where the TCL's temperature hysteresis was set to $\Delta T=1{ }^{\circ} \mathrm{C}$ or $\Delta T=2{ }^{\circ} \mathrm{C}$. Figures 5 and 6 illustrate the aggregate exergy destruction response for the MGV subject to the variable coolant pump operating speed and hysteresis temperature control in addition to the differences between the training and testing data-sets. Similarly, the dissimilar thermal response of the components which arises from the differences within the training or testing data-sets are provided in Figure 7 through Figure 8. The tabulated aggregate exergy destruction resulting from either the training or testing data-sets are formally provided in Table 1. Similarly, the final State of Charge (SoC) of the vehicle at the end of the simulation is also formally provided in Table 2.

Figure 5 through Figure 6 illustrates the differences between the tabulated exergy destruction rate resulting from the variable actuation of the TCL. The primary features which are observable includes the differences between the mission load which can be observed periodically within the mobility maneuvers, or during the reduced ancilliary load operation. The periodic mission loads during the mobility maneuvers are larger for the training data-sets versus the testing data-sets. Conversely, the mission loads during the reduced ancilliary load operation was larger for the testing data-set than the training data-set. We can also identify the periods of time in which the TCL becomes active, i.e., noticeable deviation from the nominal exergy destruction rate, in which many of the trajectories appears to track together despite the differences within the control of the TCL.

Another discernible feature which distinguishes the different exergy destruction rates of each data-set includes the point in which the TCL becomes active and the approximate amount of exergy being destroyed as a result of the TCL operation. Generally speaking, with larger temperature hysteresis $\left(\Delta T=2{ }^{\circ} \mathrm{C}\right)$, the coolant loop must remain active for longer periods of time. Conversely, a smaller temperature hysteresis $\left(\Delta T=1^{\circ} \mathrm{C}\right)$ means the coolant loop is less likely to be active for long periods of time. The TCL is also affected by the coolant pump's angular frequency. Lower angular frequencies $(\omega=250 \mathrm{rpm})$ lead to less mass flow rate. This reduces the heat transfer rate, which in turn changes the operation of the chiller, meaning we expect our exergy destruction to decrease in magnitude. 
However, the duration in which the TCL must remain active to achieve the desired cooling rate, this is expected to increase. In contrast for larger angular frequencies $(\omega=500 \mathrm{rpm})$, greater mass flow rate means more heat may be transferred away from the fluid decreasing the likelihood the TCL remains active for longer periods of times. Increased angular frequency results in a larger amount of exergy being destroyed to operate the components. The aggregate exergy destroyed as a result of the different training or testing data-sets is shown in Table 1. It is also worth mentioning that the amount of exergy being destroyed is directly tied to the state of charge of the battery, therefore if greater exergy is being destroyed as a result of the variations of the mission loads, drive cycle, and the TCL control, we expect greater energy storage utilization. More of the batteries amp-hour capacity is subsequently dissipated, which can be observed upon inspection of Table 2 .
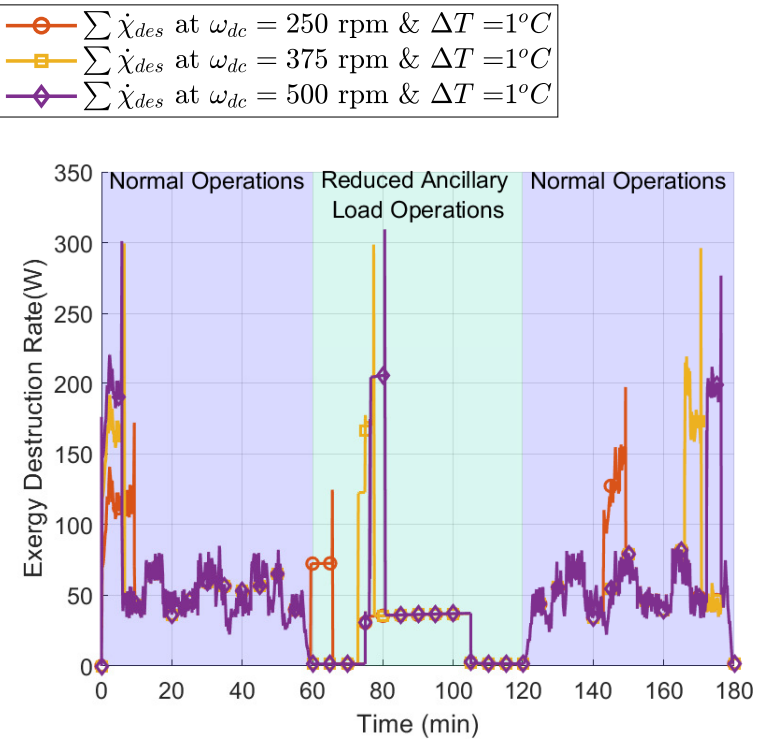

(a)
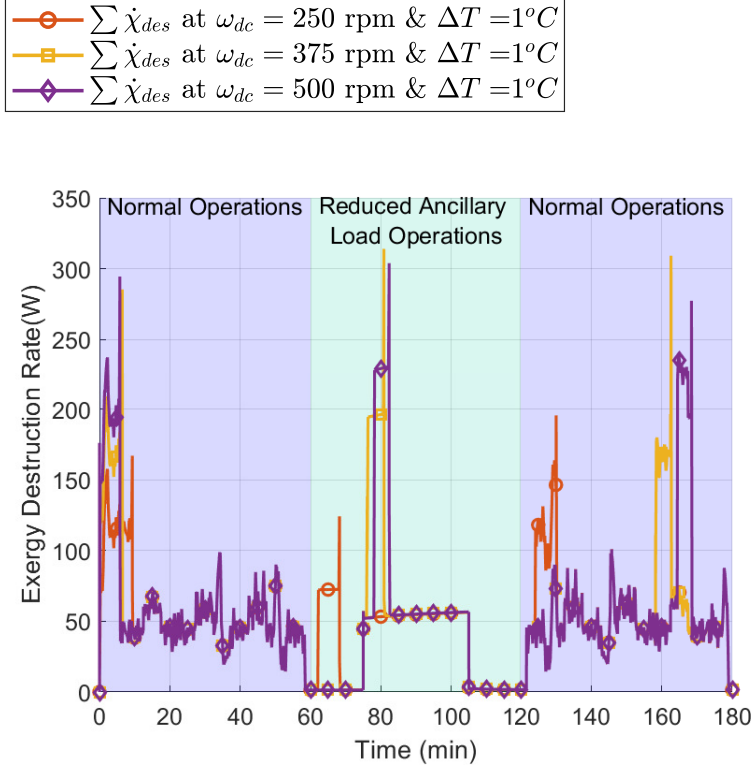

(b)

Figure 5. The exergy destruction for the MGV subject to a temperature hysteresis control of $\Delta T=1^{\circ} \mathrm{C}$ for the training data set (a) and the testing data set (b). 
$\begin{aligned}-\sum \dot{\chi}_{d e s} \text { at } \omega_{d c} & =250 \mathrm{rpm} \& \Delta T=2^{\circ} C \\ -\sum \dot{\chi}_{d e s} \text { at } \omega_{d c} & =375 \mathrm{rpm} \& \Delta T=2^{\circ} C \\ -\sum \dot{\chi}_{d e s} \text { at } \omega_{d c} & =500 \mathrm{rpm} \& \Delta T=2^{\circ} C\end{aligned}$

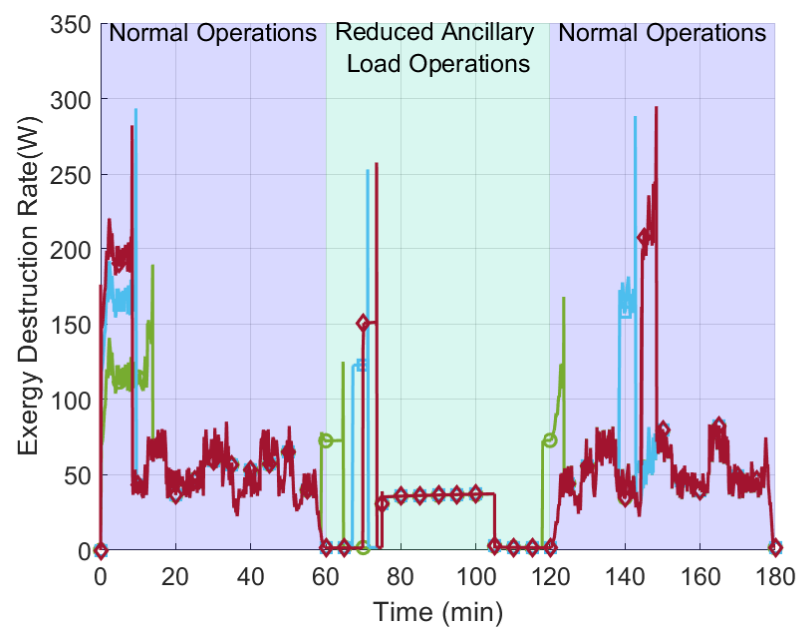

(a)

$\odot-\sum \dot{\chi}_{d e s}$ at $\omega_{d c}=250 \mathrm{rpm} \& \Delta T=2^{\circ} \mathrm{C}$

$\square-\sum \dot{\chi}_{d e s}$ at $\omega_{d c}=375 \mathrm{rpm} \& \Delta T=2^{\circ} \mathrm{C}$

$\leadsto-\sum \dot{\chi}_{d e s}$ at $\omega_{d c}=500 \mathrm{rpm} \& \Delta T=2^{\circ} C$

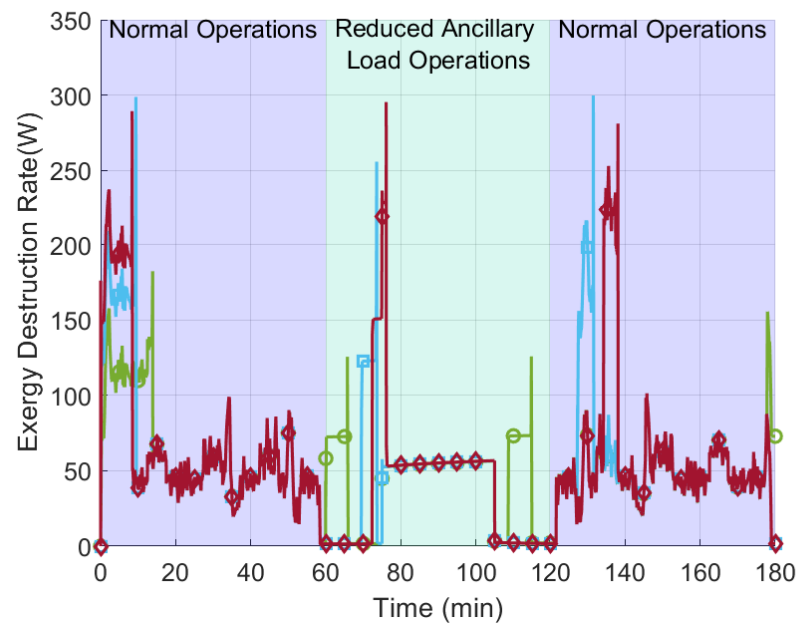

(b)

Figure 6. The exergy destruction for the MGV subject to a temperature hysteresis control of $\Delta T=2{ }^{\circ} \mathrm{C}$ for the training data set (a) and the testing data set (b). 
- $321(1)-\mathrm{T}(\mathrm{t})$ at $\omega_{d c}=250 \mathrm{rpm} \& \Delta T=1^{\circ} \mathrm{C}-0-500(1)-\mathrm{T}(\mathrm{t})$ at $\omega_{d c}=250 \mathrm{rpm} \& \Delta T=1^{\circ} \mathrm{C}$

- 321(1) - $\mathrm{T}(\mathrm{t})$ at $\omega_{d c}=375 \mathrm{rpm} \& \Delta T=1^{\circ} \mathrm{C}$ - $\mathrm{日}-500(1)-\mathrm{T}(\mathrm{t})$ at $\omega_{d c}=375 \mathrm{rpm} \& \Delta T=1^{\circ} \mathrm{C}$

- $321(1)-T(\mathrm{t})$ at $\omega_{d e}=500 \mathrm{rpm} \& \Delta T=1^{\circ} \mathrm{C}-\triangle-500(1)-\mathrm{T}(\mathrm{t})$ at $\omega_{\mathrm{de}}=500 \mathrm{rpm} \& \Delta T=1^{\circ} \mathrm{C}$

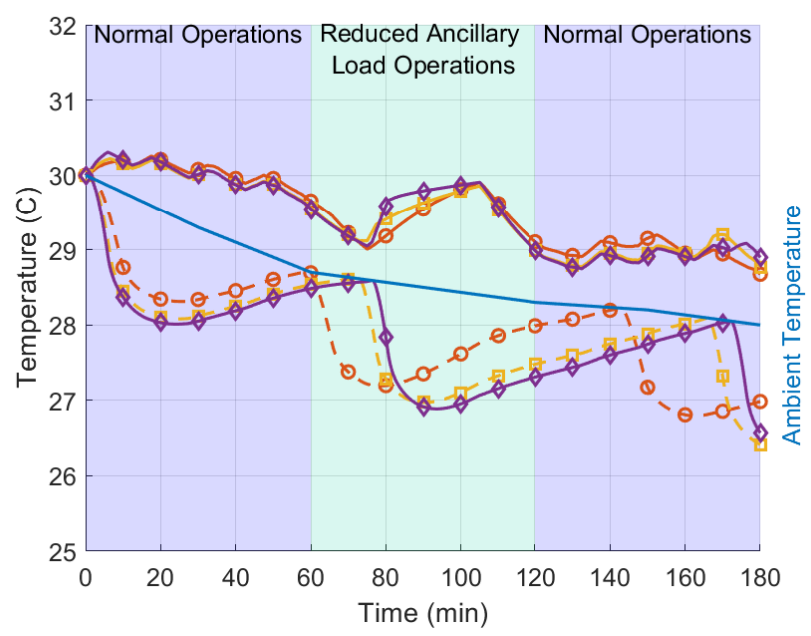

(a)

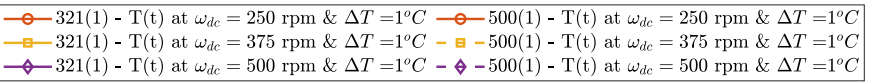

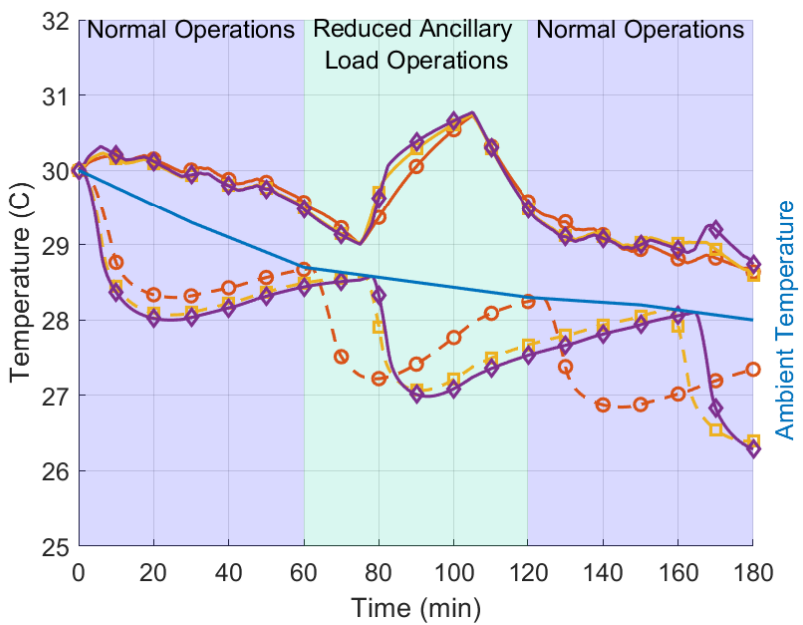

(b)

Figure 7. The thermal response of the battery 502(1) and the thermal compartment 321(1) at various coolant pump speeds and a temperature hysteresis control of $\Delta T=1{ }^{\circ} \mathrm{C}$ for the training data set (a) and testing data set $(\mathbf{b})$.

Another set of results which help to illustrate the differences between the training and testing data-sets are that of the different temperature profiles for thermal compartment and the battery's temperature as shown in Figure 7 through Figure 8. Upon inspection of these figures, we can infer that the mission loads during the reduced ancilliary load operation are larger for the testing data-set as opposed to the training data-set. This result is a direct result of a larger thermal response of the boost power converter, which is the power converter which facilitates power flow from the battery to the DC bus and all other systems of the MGV. We can also see the periods of time in which the TCL becomes active or inactive. When the TCL becomes active large and abrupt temperature changes are observed. When the TCL deactivates, then the temperature begins to rebound following a parabolic concave up trajectory at which point it seems to follow a linear trend until the temperature rises to the point in which the TCL will again become active which is a function of the TCL's temperature hysteresis. One important feature to focus in on 
within the temperature profiles is that for all training or testing data-sets shown in this section, the TCL becomes active one or two times throughout the reduced ancilliary load operation. This is the primary feature we would like to avoid, which is only possible if we can accurately characterize the dissimilar energy flows of the MGV subjective to variable time, space, or the environment.

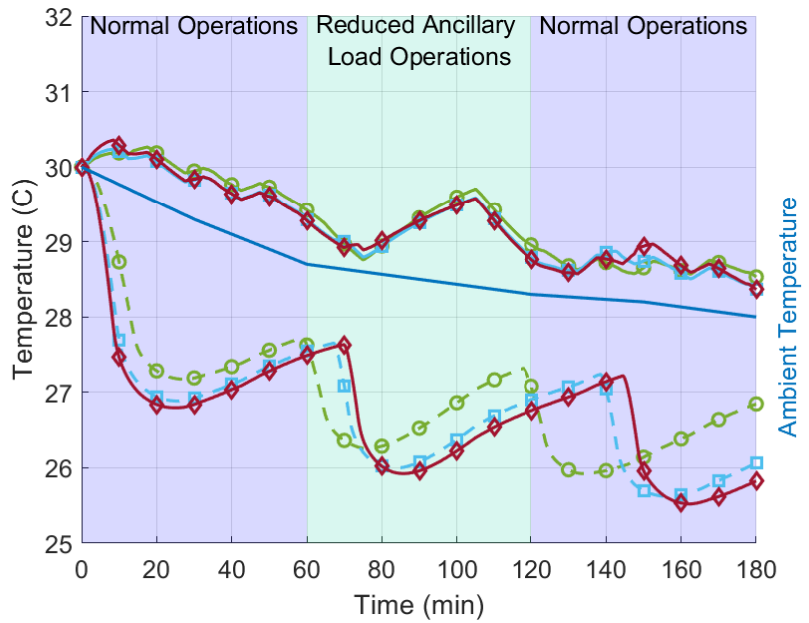

(a)

$-321(1)-\mathrm{T}(\mathrm{t})$ at $\omega_{d c}=250 \mathrm{rpm} \& \Delta T=2^{\circ} \mathrm{C}-5050(1)-\mathrm{T}(\mathrm{t})$ at $\omega_{d c}=250 \mathrm{rpm} \& \Delta T=2^{\circ} \mathrm{C}$

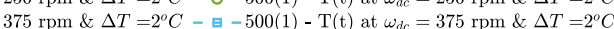

$-321(1)-\mathrm{T}(\mathrm{t})$ at $\omega_{d c}=500 \mathrm{rpm} \& \Delta T=2^{\circ} \mathrm{C}-\diamond-500(1)-\mathrm{T}(\mathrm{t})$ at $\omega_{d c}=500 \mathrm{rpm} \& \Delta T=2^{\circ} C$

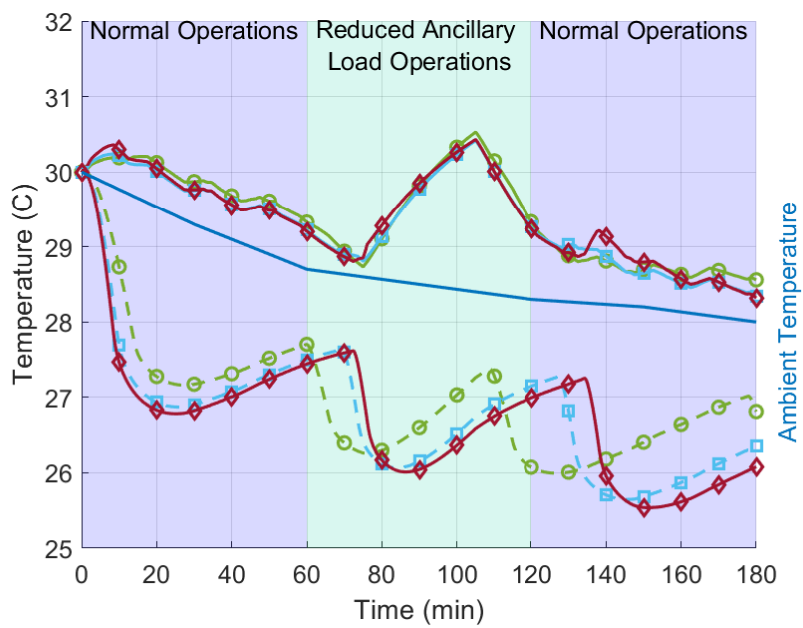

(b)

Figure 8. The thermal response of the battery 502(1) and the thermal compartment 321(1) at various coolant pump speeds and a temperature hysteresis control of $\Delta T=2{ }^{\circ} \mathrm{C}$ for the training data set (a) and testing data set (b). 
Table 1. The aggregate exergy destruction rate for each training and testing data-sets.

\begin{tabular}{ccccc}
\hline Hysteresis & Data Set & $\boldsymbol{\omega}_{d c}=\mathbf{2 5 0} \mathbf{~ r p m}$ & $\boldsymbol{\omega}_{d c}=\mathbf{3 7 5} \mathbf{~ r p m}$ & $\boldsymbol{\omega}_{d c}=\mathbf{5 0 0} \mathbf{~ r p m}$ \\
\hline \multirow{2}{*}{$\Delta T=1{ }^{\circ} \mathrm{C}$} & Train $(\mathrm{kJ})$ & 431.07 & 437.86 & 445.58 \\
\cline { 2 - 5 } & Test $(\mathrm{kJ})$ & 447.73 & 458.33 & 467.71 \\
\hline \multirow{2}{*}{$\Delta T=2{ }^{\circ} \mathrm{C}$} & Train $(\mathrm{kJ})$ & 434.51 & 438.80 & 446.53 \\
\cline { 2 - 5 } & Test $(\mathrm{kJ})$ & 453.29 & 456.44 & 467.64 \\
\hline
\end{tabular}

Table 2. The aggregate exergy destruction rate for each training and testing data-sets.

\begin{tabular}{ccccc}
\hline Hysteresis & Data Set & $\boldsymbol{\omega}_{d c}=\mathbf{2 5 0} \mathbf{~ r p m}$ & $\omega_{d c}=\mathbf{3 7 5} \mathbf{~ r p m}$ & $\boldsymbol{\omega}_{d c}=\mathbf{5 0 0} \mathbf{~ r p m}$ \\
\hline \multirow{2}{*}{$\Delta T=1{ }^{\circ} \mathrm{C}$} & Train $(\%)$ & 67.07 & 66.82 & 66.62 \\
\cline { 2 - 5 } & Test (\%) & 65.61 & 65.30 & 65.07 \\
\hline \multirow{2}{*}{$\Delta T=2{ }^{\circ} \mathrm{C}$} & Train $(\%)$ & 66.86 & 66.64 & 66.43 \\
\cline { 2 - 5 } & Test $(\%)$ & 65.24 & 65.16 & 64.91 \\
\hline
\end{tabular}

Upon completion of the twelve simulations, we used the training based data-sets for constructing our predictive algorithms. The remainder of the results are arranged as follows: Section 5.1 contains the results collected in order to observe the applicability of the predictive algorithms and Section 5.2 contains the results of the MPC-based optimization and control strategy.

\subsection{Applicability of the Predictive Algorithms}

In Jane et al. [34] we tested our predictive algorithms using multiple coolant pump angular frequencies and it showed that our predictive algorithms were insufficient to characterize the exergy and energy flow of the vehicle for the variable operating conditions tested. Our previously created predictive algorithms were all trained assuming that the coolant pump operated at $\omega_{d c}=500 \mathrm{rpm}$ and the temperature hysteresis was set to $\Delta T=2{ }^{\circ} \mathrm{C}$. When we tested our predictive capabilities we altered the coolant pumps operating frequency and the temperature hysteresis. As one might have expected, the predictive algorithms preformed poorly, leading to inaccurate energy or exergy flow characterizations. Inaccurate energy flow characterizations limited our ability to fully implement a model predictive control (MPC) strategy and improve the mission efficiency of our target platform. To improve our performance of our predictive algorithms, we utilized more training data which included alternative coolant pump operating frequencies and temperature hysteresis setpoints.

The results of the algorithms in response to two of the testing data-sets are provided in Figures 9-12. In Jane et al. [26,34] we developed multiple sets of predictive algorithms which could be used to reconcile applicable sensor measurements and reconstruct the energy and energy flow for a current point in time while using estimated information relating to the drive cycle, road grade, ambient temperature, and the mission load forecast the future energy and exergy flow. Since our ultimate goal is to improve the mission efficiency of our target platform, our results are focused on developing future energy/exergy flow characterizations. If we can provide accurate forecasts of the future energy/exergy flow considering the vehicle's future operational use, we can use the predictive algorithms to help inform a MPC-based strategy which seeks to lead to a more mission effective or efficient operation of the vehicle.

Figures 9 and 10 illustrates the response to the future forecasts of the aggregate exergy being destroyed for the differential, a power converter, and the battery. The future forecasts were generated assuming we had knowledge of the future drive cycle, ambient temperature, road grade, and mission load profile. This information was passed to a neural networks which sought to predict the aggregate load of the driven tires. This result was then fed 
into the tire neural networks which predicted the amount of destroyed exergy and stored energy, permitting the delivered energy to each drive tire to be predicted. The output of the each tire neural network then fed the differential's neural networks, which backpropagates to all other neural networks as defined by the MGV's architecture. As we can infer from Figures 9 and 10, our newly trained neural network are sufficient to characterize the dissimilar energy flows of the MGV's drive train and the only noticeable differences observed within the figures occur from 750 through $900 \mathrm{~s}$. This is one of the periodic times within the simulation that the mission load increases because we did not sufficiently alter the mission load within the training data-sets. Our predictive algorithms have a tendency to go awry during these periods.

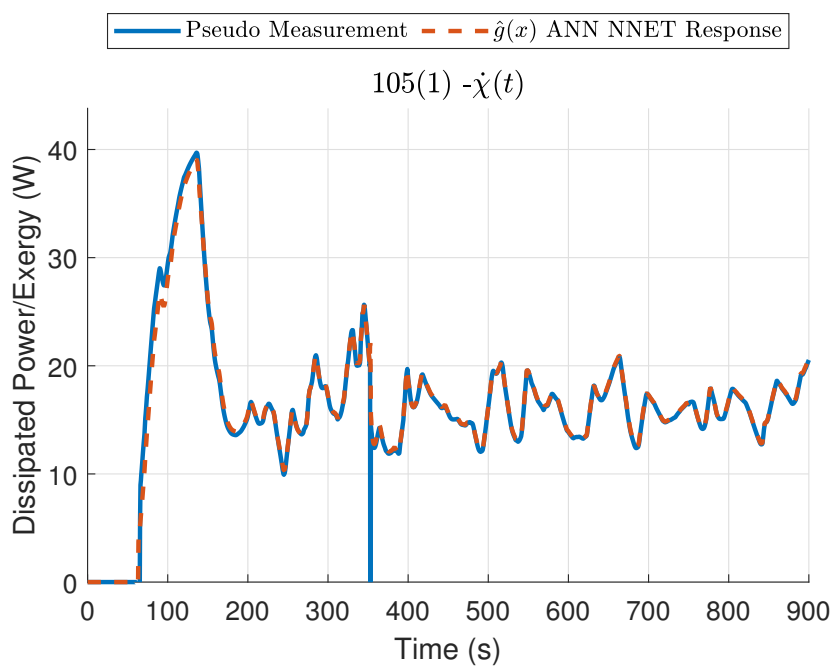

(a)

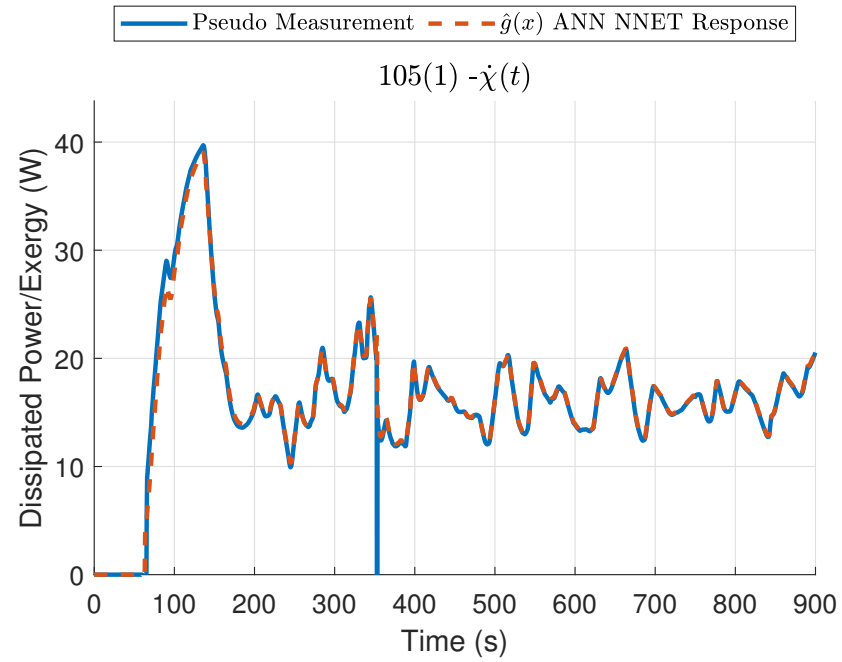

(b)

Figure 9. The future forecast of the exergy destruction rate for the differential, assuming that the coolant pump, if active would operate at $\omega_{d c}=500 \mathrm{rpm}$ and $\Delta T=2{ }^{\circ} \mathrm{C}(\mathbf{a})$ or $\omega_{d c}=250 \mathrm{rpm}$ and $\Delta T=1{ }^{\circ} \mathrm{C}(\mathbf{b})$. 


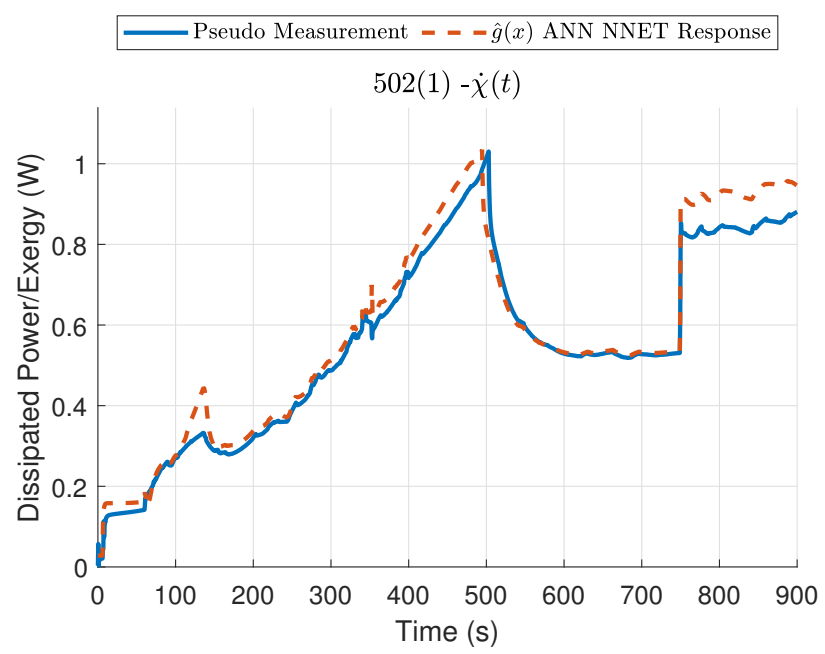

(a)

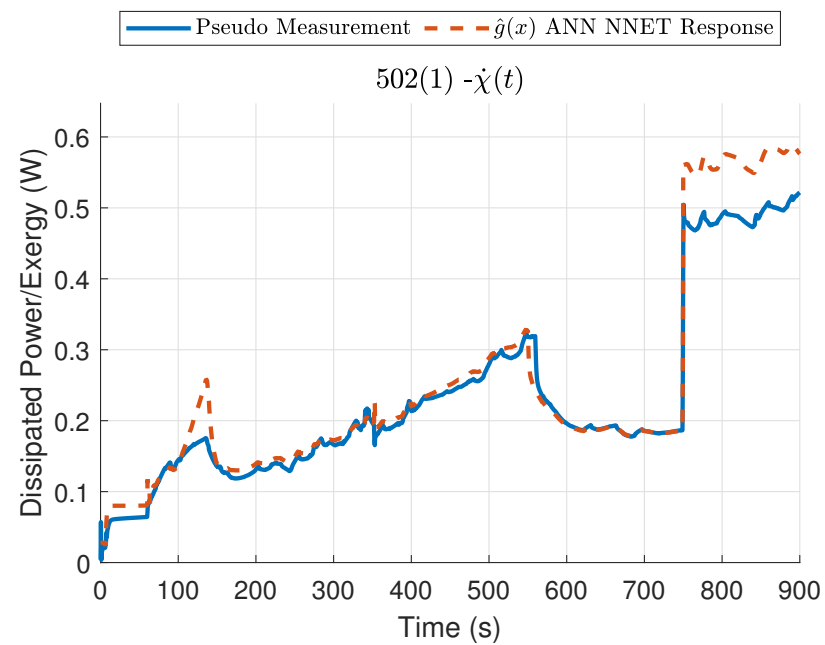

(b)

Figure 10. The future forecast of the exergy destruction rate for the battery, assuming that the coolant pump, if active would operate at $\omega_{d c}=500 \mathrm{rpm}$ and $\Delta T=2{ }^{\circ} \mathrm{C}$ (a) or $\omega_{d c}=250 \mathrm{rpm}$ and $\Delta T=1{ }^{\circ} \mathrm{C}(\mathbf{b})$.

Figures 11 and 12 illustrates the response of our predictive algorithms for the battery and the thermal compartment. These two components were selected as a result that they are the primary components which are to be impacted by the activation of the coolant loop. The coolant loop will be activated by the battery's temperature rising above the upper bound. Once the lower bound has been achieved the coolant loop will deactivate. Each electrical/mechanical component in addition to the thermal fluid components may also transfer heat to the thermal compartment, which results in an aggregate temperature response of the thermal compartment. Upon inspection of Figures 11 and 12 we see that the battery temperature response performs well for the future forecast. However, there is a noticeable difference for the thermal compartment temperature, which directly corresponds to the periods of time in which the mission load increases. A significant increase in the predicted thermal compartment temperature will lead to over predictions of the heat transfer rates between each individual component. This leads to degradation in each of the component's thermal response. Despite the noticeable degradation, it is believed that as long as the forecast horizon of the predictive algorithms do not extend sufficiently far into the future and that the increased mission load does not remain active for extended periods of time, the neural networks should continue to provide sufficiently accurate predictions such that a MPC-based algorithm can be implemented to improve mission efficiency. 


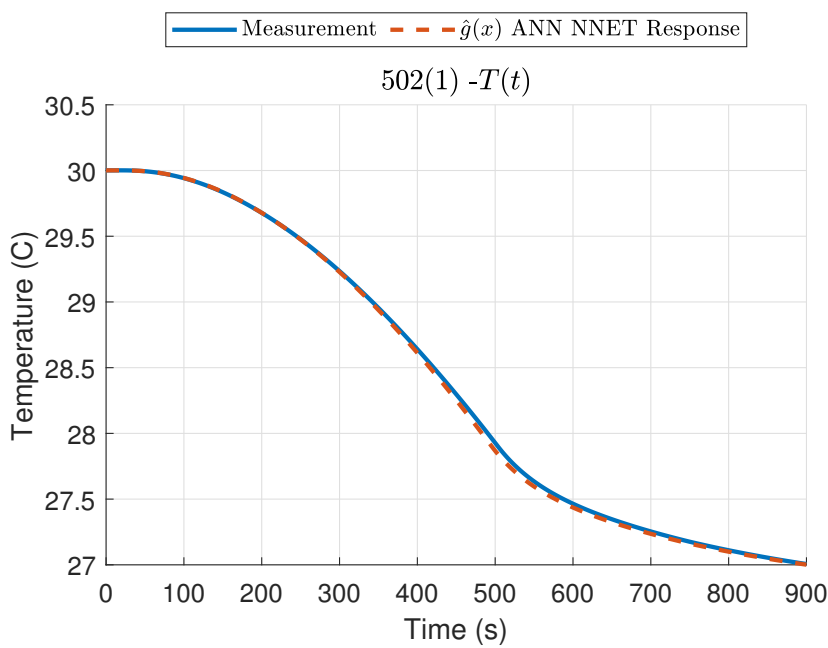

(a)

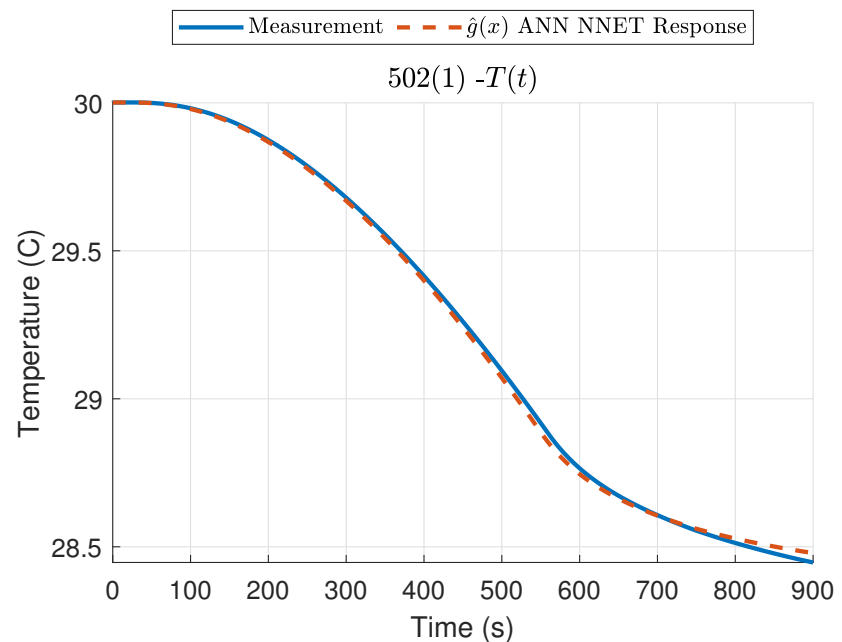

(b)

Figure 11. The future forecast of the thermal response of the battery, assuming that the coolant pump, if active would operate at $\omega_{d c}=500 \mathrm{rpm}$ and $\Delta T=2{ }^{\circ} \mathrm{C}(\mathbf{a})$ or $\omega_{d c}=250 \mathrm{rpm}$ and $\Delta T=1{ }^{\circ} \mathrm{C}(\mathbf{b})$.

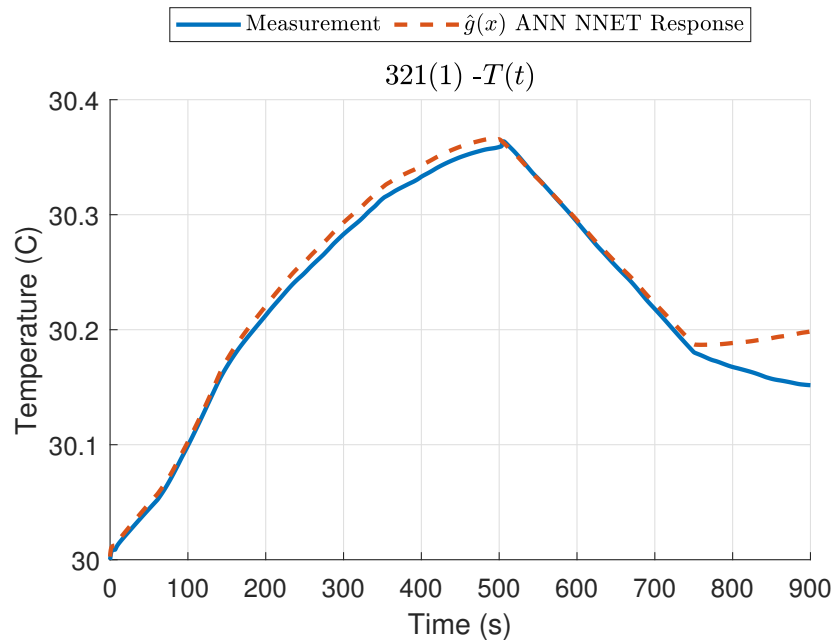

(a)

Figure 12. Cont. 


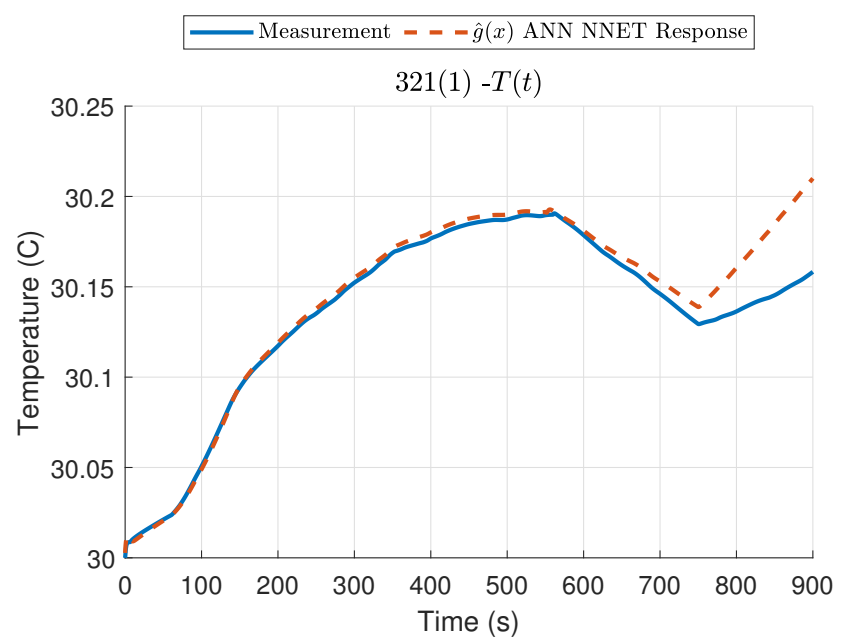

(b)

Figure 12. The future forecast of the thermal response of the thermal compartment, i.e., the compartment in which all other electrical components interact with, assuming that the coolant pump, if active would operate at $\omega_{d c}=500 \mathrm{rpm}$ and $\Delta T=2{ }^{\circ} \mathrm{C}(\mathbf{a})$ or $\omega_{d c}=250 \mathrm{rpm}$ and $\Delta T=1{ }^{\circ} \mathrm{C}(\mathbf{b})$.

\subsection{Model Predictive Control}

In this final subsection we implemented our MPC-based strategy which used multiple neural networks to predict the dissimilar energy flows in an attempt to improve mission effectiveness and efficiency of the MGV. Figure 13 compares the exergy destruction rate and thermal response for four of the six testing data-set responses when compared to simulated MGV which used the MPC-based strategy to improve mission effectiveness or efficiency.

Recall, the goal of the optimization routine was to minimize exergy destruction i.e., improve mission efficiency and improve mission effectiveness by delaying the activation of the TCL such that it would not trigger during a reduced ancilliary load operation. During a reduced ancilliary load operation, the aggregate load of the vehicle should be reduced such that no unnecessary components are activated.

Figure 13a shows the exergy destruction rate for four of the six aggregate exergy destruction response of the MGV subject to the testing data-set in addition to the aggregate exergy destruction response of the MGV in which the MPC was deployed. Similarly, Figure $13 \mathrm{~b}$ contains four of the six thermal response for the battery and the thermal compartment of the MGV subject to the testing data-set in addition to the thermal response for the battery and the thermal compartment of the MGV in which the MPC was deployed. In either figure, four of the five trajectories indicate that the TCL becomes activated during reduced ancilliary load operation, i.e., the MGV will be less effective. The only trajectory which does not trigger the TCL to become activate during the reduced ancilliary load operation is the trajectory which results from deploying the MGV's MPC based predictive algorithms. When the TCL becomes active, we see an noticeable increase in the exergy destruction rate and a characteristic deviation from what is presumed to be the nominal exergy destruction rate. Similarly if we look at the thermal response of the battery, we notice a sudden and rapid concave down trajectory. Once the TCL has sufficiently cooled the battery, the TCL becomes inactive, and the batteries thermal response will follow a concave up trajectory and naturally decay to more of linear trend increase in temperature as time progresses.

Analysis of both the exergy destruction rate and the thermal response of the optimal trajectory indicates that the TCL remains active for a long period of time during the first mobility maneuver. This is to sufficiently cool the battery such that the TCL will not need to be activated until after the reduced ancilliary load mission has passed. In this way the MPC was able to identify a set of angular frequency operating points and temperature delta setpoints that permitted the MGV to operate more effectively. Interestingly, as a result of the MPC, the MGV now destroyed $461.31 \mathrm{~kJ}$ of exergy and consumed $34.92 \%$ of 
the energy contained within the MGV's battery. The MGV consumed less percentage of the total energy storage capacity and destroyed less exergy than the MGV when the TCL operated at $\omega_{d c}=500 \mathrm{rpm}$ and $\Delta T=1{ }^{\circ} \mathrm{C}$ or $2{ }^{\circ} \mathrm{C}$, but was less efficient when the TCL operated at $\omega_{d c}=375 \mathrm{rpm}$ and $\Delta T=1{ }^{\circ} \mathrm{C}$ or $2{ }^{\circ} \mathrm{C}$. The MPC was effectively able to identify a potential set of operating points that saved some energy, but more importantly allowed the vehicle to operate more effectively in the presence of reduced ancilliary load operation.

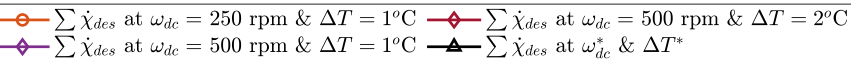

$\begin{aligned}-\sum \dot{\chi}_{\text {des }} \text { at } \omega_{d c} & =500 \mathrm{rpm} \& \Delta T=1^{\circ} \mathrm{C} \\ -\sum \dot{\chi}_{d e s} \text { at } \omega_{d c} & =250 \mathrm{rpm} \& \Delta T=2^{\circ} \mathrm{C}\end{aligned}$

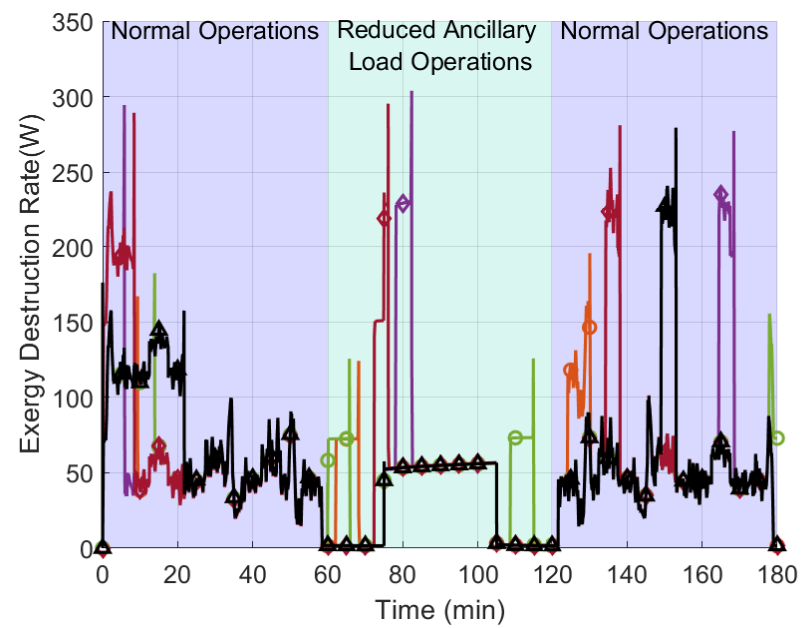

(a)

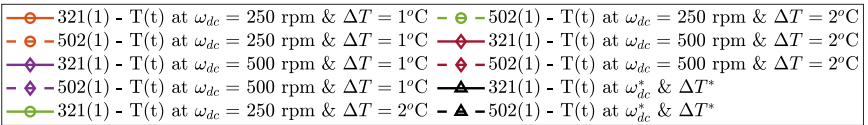

$-\diamond-502(1)-\mathrm{T}(\mathrm{t})$ at $\omega_{d c}=500 \mathrm{rpm} \& \Delta T=1^{\circ} \mathrm{C}-321(1)-\mathrm{T}(\mathrm{t})$ at $\omega_{d c}^{*} \& \Delta T$
$-321(1)-\mathrm{T}(\mathrm{t})$ at $\omega_{d c}=250 \mathrm{rpm} \& \Delta T=2^{\circ} \mathrm{C}-\mathbf{\Delta}-502(1)-\mathrm{T}(\mathrm{t})$ at $\omega_{d c}^{*} \& \Delta T^{*}$

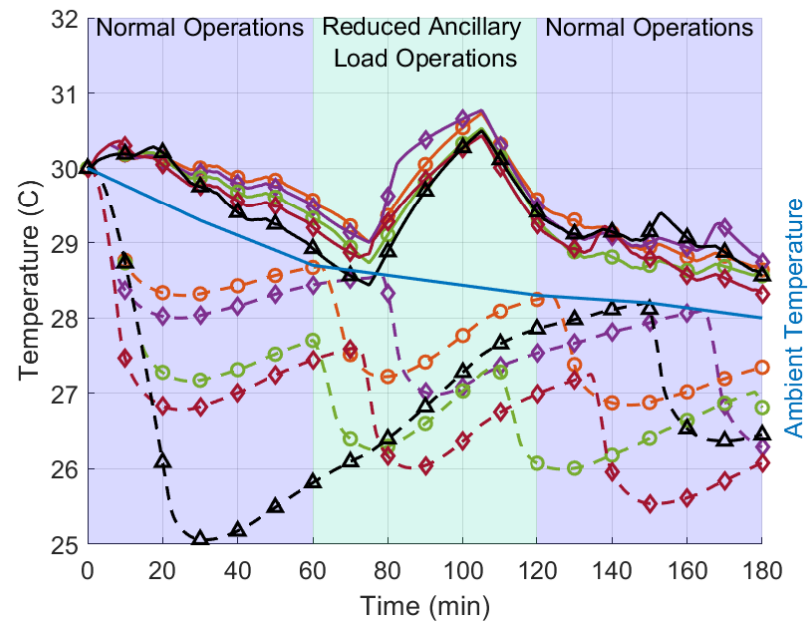

(b)

Figure 13. The exergy destruction response (a) and the thermal response of the battery 502(1) and the thermal compartment 321(1) for four of the six operating set-points for the TCL when compared to the MPC's more effective solution as identified from the predictive algorithms (b). 
Our MPC was executed twice throughout the total simulation epoch, once at the beginning, and once after fifty-five minutes after the simulation began. During the first simulation epoch the MPC identified the optimal coolant pump angular velocity $\omega_{d c}^{*}$ and hysteresis delta temperature $\Delta T^{*}$ to be that of Equation (22).

$$
\left[\begin{array}{c}
\omega_{d c}^{*} \\
\Delta T^{*} \\
t^{*}
\end{array}\right]=\left[\begin{array}{cccc}
375 & 375 & 500 & 500 \\
4 & 4 & 1 & 1 \\
0 & 3300 & 3300.5 & 10800
\end{array}\right]
$$

At the next optimization interval, the MPC's solution did not converge which would indicate that none of the unique one-hundred and forty-four possible solutions for the remaining simulation epoch could ensure a more mission effective operation of the MGV. In this case the MPC algorithm used the previously found optimal solution to create a new theoretical optimal solution candidate as shown in Equation (23).

$$
\left[\begin{array}{c}
\omega_{d c}^{*} \\
\Delta T^{*} \\
t^{*}
\end{array}\right]=\left[\begin{array}{cccc}
500 & 500 & 500 & 500 \\
1 & 1 & 1 & 1 \\
3300 & 6900 & 6900.5 & 10800
\end{array}\right]
$$

It is unclear how this happens but a likely cause is that upon updating the memory and states of the model, the neural network model over or under estimates some of the dissimilar energy flows which impact the thermal behavior or heat transfer characteristics of the system. These inaccurate predictions then lead the neural networks to under perform. Recall that in Section 5.1, we briefly explored the applicability of the predictive algorithms and our analysis indicated good agreement between the neural network performance and the simulated response for a fifteen-minute epoch. However near the end of the fifteen-minute epoch there was a noticeable deviation of the batteries thermal temperature response, in part due to the inclusion of mission load. When the MPC was run, it was predicting the exergy flow and temperature responses for two to three hours in the future. Longer simulation epochs are better for ensuring we can identify the optimal energy/exergy solution which is more effective, however, it presents more avenues for the predictive algorithms to drift. Over or under predictions are more likely to occur as a result of states or memories deviating substantially from any known conditions. In the presence of regular feedback the neural networks performance is less likely to degrade. This establishes an important result that the applicability of the predictive algorithms must not exceed some predefined forecast horizon. The algorithms may only be good for a small forecast horizon; larger forecast horizons can lead to degraded forecasts and the inability to optimize the performance of the target platform.

\section{Conclusions and Future Work}

We presented an exergy based model predictive control (MPC) method for battlefield analysis and control. Using MATLAB/Simulink in combination with Simscape's thermal, thermal-fluid, and mechanical tool-boxes, we created a simulation architecture in which we could simulate the dynamics of a MGV and (1) simultaneously run our neural networkbased predictive algorithms and compare to the simulated truth model, or (2) run our MPC to optimize the vehicle's performance. Our results indicated the predictive algorithms showed good agreement with the simulated truth model indicating that the algorithms were likely sufficient for implementing a MPC based optimization routine. The predictive algorithms were trained on multiple training data-sets and tested on alternative data-sets using supervised learning practices.

Once we had determined that the predictive algorithms seemed to sufficiently predict the dissimilar energy flows and the thermal response, we implemented a brute-force optimization routine to improve the effectiveness of the vehicle, while maximizing the efficiency of the vehicle. The initial execution of the MPC yielded a candidate solution which led to increased effectiveness and efficiency of the MGV when compared to the 
previously simulated training or testing data sets. Unfortunately, the subsequent executions of the MPC were inconclusive, i.e., the MPC was unable to identify a candidate solution which could increase effectiveness and efficiency of the vehicle. The likely reason is the updates of the memory and states of the predictive algorithms lead to under and over estimations of some of the dissimilar energy flows which lead to under or over predictions of the heat transfer response. This leads to poor temperature response predictions. It is also possible that because the first operating point identified by the MPC sufficiently deviated from the training data-sets and upon updating of the memory or states of the model, the new operational space sufficiently deviated from the training space and led to degradation of the predictive algorithms.

The work presented indicates that it is possible to create a set of artificial neural networks which can be used for developing timeseries predictions of dissimilar energy flows and thermal responses with sufficient accuracy that an MPC can be deployed to increase mission effectiveness or efficiency of a target system. The only caveat to this is the response of the predictive algorithms may degrade for longer forecast horizons, and thus it becomes paramount to ensure that the predictive algorithms developed are not overextended beyond the reasonable forecast horizon. For future work, we intended to continue to develop and deploy dissimilar types of artificial neural networks for energy flow and temperature prediction and improve the algorithms developed. We intend to focus on larger Army relevant platforms which include internal combustion engines [35] including squad multipurpose equipment transport (SMET). Further we intend to improve the optimization routing by integrating more standard and efficient optimization based algorithms which further improves the ability to utilize the predictive algorithms for improving performance of a target platform. We are also beginning to explore developing and deploying these same type of predictive algorithms using real-time online training capabilities in combination with unsupervised training such that we can adaptability and flexibly train the algorithms to improve efficiency or mission effectiveness of a target platform based on the defined operational scenario.

Author Contributions: Author contributions for this work are as follows: Conceptualization, R.J. and C.J.; methodology, R.J.; software, R.J. and T.Y.K.; validation, R.J., T.Y.K. and C.J.; formal analysis, R.J.; investigation, R.J., T.Y.K. and C.J.; resources, R.J. and C.J.; writing-original draft preparation, R.J., E.G., E.M. and C.J.; writing-review and editing, R.J., E.G., E.M. and C.J.; visualization, R.J., T.Y.K. and C.J.; supervision, C.J.; project administration, C.J.; funding acquisition, C.J. All authors have read and agreed to the published version of the manuscript.

Funding: This research received no external funding.

Institutional Review Board Statement: Not applicable.

Informed Consent Statement: Not applicable.

Acknowledgments: The authors would like to gratefully acknowledge the support of Combat Capabilities Development Command (CCDC), U.S. Army Research Laboratory (ARL), Sensors and Electronic Devices Directorate (SEDD). The appearance of U.S. Department of Defense (DoD) visual information does not imply or constitute DoD endorsement.

Conflicts of Interest: The authors declare no conflict of interest.

\section{Abbreviations}

The following abbreviations are used in this manuscript:

AI

AlexNet

ANN

C5

CA

$\mathrm{CNN}$
Artificial Intelligence

Alex Krizhevsky's Neural Network Architecture

Artificial Neural Network

Cargo Five

Constant Acceleration

Convolution Neural Network 


\begin{tabular}{|c|c|}
\hline $\mathrm{COP}$ & Coefficient of Performance \\
\hline CS & Constant Speed \\
\hline CVI & Chemical Vapor Infiltration \\
\hline DC & Direct Current \\
\hline DNN & Deep Neural Network \\
\hline DropCNN & Drop Convolution Neural Network \\
\hline F1 & Function One \\
\hline FLT & First Law of Thermodynamics \\
\hline G-TL & Gas and Thermal Liquid \\
\hline GFE & Government Furnished Equipment \\
\hline GPS & Global Positioning Satellite \\
\hline $\mathrm{H}_{\infty}$ & Hardy Infinity \\
\hline JF & Joint Fine-tuning \\
\hline LSTM or LSTMN & Long Short Term Memory or Long Short Term Memory Network \\
\hline M1-A1 & Model One - Advancement One \\
\hline MGV & Mobile Ground Vehicle \\
\hline MM & Markov-based Models \\
\hline ML & Machine Learning \\
\hline MLP & Multi-Layer Preceptron \\
\hline MPC & Model Predictive Control \\
\hline MDO & Multi-Domain Operations \\
\hline NN or NNET & Neural Network \\
\hline PHEB & Plug-n Hybrid Electric Bus \\
\hline RAF & Random Forest \\
\hline RBF & Radial Basis Function \\
\hline RMSE & Root Means Squared Error \\
\hline RNN & Recurrent Neural Network \\
\hline RRt & Rapidly-exploring Random Tree \\
\hline ResNet & Residual Neural Network \\
\hline ResNet50 & Residual Neural Network Fifty \\
\hline RPM & Revolutions Per Minute \\
\hline SANN & Shallow Artificial Neural Network \\
\hline SMET & Squad Multipurpose Equipment Transport \\
\hline SLT & Second Law of Thermodynamics \\
\hline SoC & Sate of Charge \\
\hline TL & Thermal Liquid \\
\hline TMS & Thermal Management System \\
\hline TCL & Thermal Coolant Loop \\
\hline VGG16 & Visual Geometry Group's Sixteenth Neural Network Architecture \\
\hline VGG19 & Visual Geometry Group's Nineteenth Neural Network Architecture \\
\hline
\end{tabular}

\section{Appendix A}

The intent of this section is to provide insight regarding our predictive algorithms. Appendix A.1 a table that contains list of unique identification number at its relation to the MGV. It also points to multiple tables as defined in Appendix A.2 help to describe the input and target features used to develop the SANNs and the LSTMs.

\section{Appendix A.1}

This Appendix contains list of unique identification number for each asset as shown in Figure 2, provided in Table A1. The information as provided within the table will help to distinguish the difference between each asset and be used to define the differences within the neural network architectures provided within subsequent appendices subsections. 
Table A1. The mobile ground vehicle's (MGV) asset list and their corresponding unique identification number, and the table in which the neural networks primary configuration features are defined.

\begin{tabular}{lcc}
\hline Asset & Identification Number & NNet Configuration \\
\hline Rear Differential & $105(1)$ & Table A3 \\
\hline Right Rear Tire & $106(1)$ & Table A4 \\
\hline Left Rear Tire & $106(2)$ & Table A5 \\
\hline Right Front Tire & $106(3)$ & Table A6 \\
\hline Left Front Tire & $106(4)$ & Table A7 \\
\hline Load & $107(1)$ & - \\
\hline Boost Power Converter & $205(1)$ & Table A8 \\
\hline Buck Power Converter & $205(2)$ & Table A9 \\
\hline Buck Power Converter & $205(3)$ & Table A10 \\
\hline DC Bus & $207(1)$ & - \\
\hline Cold Plate & $305(1)$ & Table A11 \\
\hline Coolant Pump & $315(1)$ & Table A12 \\
\hline Thermal Compartment & $321(1)$ & Table A13 \\
\hline Coolant Tank & $322(1)$ & Table A14 \\
\hline Chiller & $323(1)$ & Table A15 \\
\hline DC Motor & $400(1)$ & Table A16 \\
\hline DC Motor & $400(2)$ & Table A17 \\
\hline Battery & $502(1)$ & Table A18 \\
\hline
\end{tabular}

\section{Appendix A.2}

This Appendix contains a compressive list which defines the sensor list in combination with a symbol used to abbreviate the sensor value as shown in Table A2. These abbreviations will be used to describe the neural networks. We developed multiple sets of Shallow Artificial Neural Network (SANN) and Long Short Term Memory Network (LSTMNs) and used them for (1) reconcile past state information not directly observable from the available sensor measurements and (2) to characterize the future energy/exergy flow behavior of the asset. Neural networks used to reconcile past state information will be classified as state estimator, conversely neural networks used to forecast or predict future energy/exergy flow behavior will be classified as state predictor. Additional subscripts may be added to symbols as shown in Table A2, this is done to distinguish between a different engineering domain, as an example $m$ or mech mechanical, $e$ or elec electrical, th for thermal, or $t f$ for thermal fluid. 
Table A2. The mobile ground vehicle's (MGV) sensor list and the corresponding symbol used to abbreviate the sensor value.

\begin{tabular}{|c|c|}
\hline Signal & Symbol \\
\hline Mission Load Profile & $I_{m i s}$ \\
\hline Reference Mission Load Profile & $I_{m i s, r}$ \\
\hline Source Current & $I_{s r c}$ \\
\hline Source Voltage & $V_{s r c}$ \\
\hline Load Current & $I_{\text {lod }}$ \\
\hline Load Voltage & $V_{\text {lod }}$ \\
\hline Source Angular Velocity & $\omega_{s r c}$ \\
\hline Source Torque & $\tau_{s r c}$ \\
\hline Load Angular Velocity & $\omega_{\text {lod }}$ \\
\hline Load Torque & $\tau_{l o d}$ \\
\hline Steady State Reference Speed & $S S_{m, r}$ \\
\hline Angular Velocity of DC Motor & $\omega_{m, s r c}$ \\
\hline Source Power & $P_{s r c}$ \\
\hline Load Power & $P_{l o d}$ \\
\hline Dissipated Power & $P_{\text {loss }}$ \\
\hline Stored Power & $P_{s t r}$ \\
\hline Component Temperature & $T_{\text {th }}$ \\
\hline Battery Temperature & $T_{\text {th,batt }}$ \\
\hline Thermal Compartment Temperature & $T_{\text {th,ext }}$ \\
\hline Ambient Temperature & $T_{t h, a m b}$ \\
\hline Reference Ambient Temperature & $T_{t h, a m b, r}$ \\
\hline Cold Plate Temperature & $T_{t h, c p}$ \\
\hline Thermal Fluid Source Power & $\phi_{t f, s r c}$ \\
\hline Thermal Fluid Source Pressure & $\Psi_{t f, s r c}$ \\
\hline Thermal Fluid Source Temperature & $T_{t f, s r c}$ \\
\hline Thermal Fluid Source Mass Flow Rate & $\dot{m}_{t f, s r c}$ \\
\hline Thermal Fluid Load Phi & $\phi_{t f, l o d}$ \\
\hline Thermal Fluid Load Pressure & $\Psi_{t f, l o d}$ \\
\hline Thermal Fluid Load Temperature & $T_{t f, l o d}$ \\
\hline Thermal Fluid Load Mass Flow Rate & $\dot{m}_{t f, l o d}$ \\
\hline Longitudinal Velocity & $V_{x}$ \\
\hline Reference Longitudinal Velocity & $V_{x, r}$ \\
\hline Longitudinal Acceleration & $A_{x}$ \\
\hline Reference Longitudinal Acceleration & $A_{x, r}$ \\
\hline Wind Velocity & $W_{w}$ \\
\hline Reference Wind Velocity & $W_{w, r}$ \\
\hline Heat Transfer to Ambient Conditions & $P_{t h, r e j}$ \\
\hline Heat Transfer to Thermal Compartment & $P_{t h, t r a n}$ \\
\hline Heat Transfer through Conduction & $P_{\text {th, cond }}$ \\
\hline Heat Transfer through Convection & $P_{t h, \text { conv }}$ \\
\hline Exergy Destruction Rate & $\dot{\chi}$ \\
\hline Binary Relay State (On/Off) & $\Xi$ \\
\hline Measure of Time Relay has been Active & $\tau_{o n}$ \\
\hline Measure of Time Relay has been Inactive & $\tau_{o f f}$ \\
\hline
\end{tabular}


Table A3. The rear differential 105(1) neural network configurations.

\begin{tabular}{|c|c|c|c|c|}
\hline Input Features & $\begin{array}{l}\text { Output } \\
\text { Features }\end{array}$ & $\begin{array}{l}\text { SANN Hidden } \\
\text { Layer Size }\end{array}$ & $\begin{array}{l}\text { LSTMN Hidden } \\
\text { Layer Size }\end{array}$ & Purpose \\
\hline $\begin{array}{l}P_{m, s r c}, P_{m, l o d, \text { right }}, \quad P_{m, l o d, l e f t}, P_{m, s r c}- \\
\left(P_{m, l o d, r i g h t}+P_{m, l o d, l e f t}\right) \\
P_{m, s r c}, P_{m, l o d, \text { right }}, P_{m, l o d, l e f t}, P_{m, s r c}- \\
\left(P_{m, l o d, \text { right }}+P_{m, l o d, l e f t}\right)\end{array}$ & $\begin{array}{l}P_{\text {loss }} \\
\dot{\chi}_{\text {mech }}\end{array}$ & 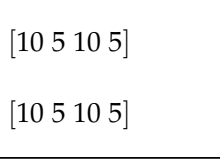 & {$[25]$} & Estimator \\
\hline $\begin{array}{l}P_{m, l o d, r i g h t}, P_{m, l o d, l e f t}, V_{x, r},\left(P_{m, l o d, r i g h t}+\right. \\
\left.P_{m, l o d, l e f t}\right) \\
P_{m, l o d, r i g h t}, P_{m, l o d, l e f t}, V_{x, r},\left(P_{m, l o d, r i g h t}+\right. \\
\left.P_{m, l o d, l e f t}\right) \\
P_{m, l o d, r i g h t}, P_{m, l o d, l e f t}, V_{x, r},\left(P_{m, l o d, r i g h t}+\right. \\
\left.P_{m, l o d, l e f t}\right)\end{array}$ & $\begin{array}{l}P_{m, s r c} \\
P_{l o s s}\end{array}$ & $\begin{array}{l}{\left[\begin{array}{lllll}5 & 25 & 15 & 10 & 5\end{array}\right]} \\
{\left[\begin{array}{lllll}5 & 25 & 15 & 10 & 5\end{array}\right]} \\
{\left[\begin{array}{lllll}5 & 25 & 15 & 10 & 5\end{array}\right]}\end{array}$ & {$[25]$} & Predictor \\
\hline
\end{tabular}

Table A4. The right rear tire 106(1) neural network configurations.

\begin{tabular}{|c|c|c|c|c|}
\hline Input Features & $\begin{array}{l}\text { Output } \\
\text { Features }\end{array}$ & $\begin{array}{l}\text { SANN Hidden } \\
\text { Layer Size }\end{array}$ & $\begin{array}{l}\text { LSTMN Hidden } \\
\text { Layer Size }\end{array}$ & Purpose \\
\hline $\begin{array}{l}P_{m, s r c}, P_{m, l o d} \\
\tau_{m, s r c}, \omega_{m, s r c}, \omega_{m, l o d} \\
P_{m, s r c}, P_{m, l o d}\end{array}$ & $\begin{array}{l}P_{\text {loss }} \\
P_{\text {m,lod }} \\
\dot{\chi}_{\text {mech }}\end{array}$ & $\begin{array}{l}{\left[\begin{array}{ll}10 & 5\end{array}\right]} \\
{\left[\begin{array}{ll}10 & 5\end{array}\right]} \\
{\left[\begin{array}{ll}10 & 5\end{array}\right]}\end{array}$ & {$[25]$} & Estimator \\
\hline $\begin{array}{l}P_{m, l o d}, V_{x, r} \\
P_{m, l o d}, V_{x, r} \\
P_{m, l o d}, V_{x, r}\end{array}$ & $\begin{array}{l}P_{m, s r c} \\
P_{\text {loss }} \\
\dot{\chi}_{\text {mech }}\end{array}$ & $\begin{array}{l}{\left[\begin{array}{llll}5 & 15 & 10 & 5\end{array}\right]} \\
{\left[\begin{array}{llll}5 & 15 & 10 & 5\end{array}\right]} \\
{\left[\begin{array}{llll}5 & 15 & 10 & 5\end{array}\right]}\end{array}$ & {$[25]$} & Predictor \\
\hline
\end{tabular}

Table A5. The left rear tire 106(2) neural network configurations.

\begin{tabular}{|c|c|c|c|c|}
\hline Input Features & $\begin{array}{l}\text { Output } \\
\text { Features }\end{array}$ & $\begin{array}{l}\text { SANN Hidden } \\
\text { Layer Size }\end{array}$ & $\begin{array}{l}\text { LSTMN Hidden } \\
\text { Layer Size }\end{array}$ & Purpose \\
\hline$P_{m, s r c}, P_{m, l o d}$ & $P_{\text {loss }}$ & {$\left[\begin{array}{ll}10 & 5\end{array}\right]$} & \multirow{3}{*}[25]{} & \multirow{3}{*}{ Estimator } \\
\hline$\tau_{m, s r c}, \omega_{m, s r c}, \omega_{m, l o d}$ & $P_{m, l o d}$ & {$\left[\begin{array}{ll}10 & 5\end{array}\right]$} & & \\
\hline$P_{m, s r c}, P_{m, l o d}$ & $\dot{\chi}_{m e c h}$ & {$\left[\begin{array}{ll}10 & 5\end{array}\right]$} & & \\
\hline$P_{m, l o d}, V_{x, r}$ & $P_{m, s r c}$ & {$\left[\begin{array}{llll}5 & 15 & 10 & 5\end{array}\right]$} & \multirow{3}{*}[25]{} & \multirow{3}{*}{ Predictor } \\
\hline$P_{m, l o d}, V_{x, r}$ & $P_{\text {loss }}$ & {$\left[\begin{array}{llll}5 & 15 & 10 & 5\end{array}\right]$} & & \\
\hline$P_{m, l o d}, V_{x, r}$ & $\dot{\chi}_{m e c h}$ & {$\left[\begin{array}{llll}5 & 15 & 10 & 5\end{array}\right]$} & & \\
\hline
\end{tabular}

Table A6. The right front tire 106(3) neural network configurations.

\begin{tabular}{|c|c|c|c|c|}
\hline Input Features & $\begin{array}{l}\text { Output } \\
\text { Features }\end{array}$ & $\begin{array}{l}\text { SANN Hidden } \\
\text { Layer Size }\end{array}$ & $\begin{array}{l}\text { LSTMN Hidden } \\
\text { Layer Size }\end{array}$ & Purpose \\
\hline$P_{m, s r c}, P_{m, l o d}$ & $P_{\text {loss }}$ & {$\left[\begin{array}{ll}10 & 5\end{array}\right]$} & \multirow{3}{*}[25]{} & \multirow{3}{*}{ Estimator } \\
\hline$\tau_{m, s r c}, \omega_{m, s r c}, \omega_{m, l o d}$ & $P_{m, l o d}$ & {$\left[\begin{array}{ll}10 & 5\end{array}\right]$} & & \\
\hline$P_{m, s r c}, P_{m, l o d}$ & $\dot{\chi}_{m e c h}$ & {$\left[\begin{array}{ll}10 & 5\end{array}\right]$} & & \\
\hline$P_{m, l o d}, V_{x, r}$ & $P_{m, s r c}$ & {$\left[\begin{array}{llll}5 & 15 & 10 & 5\end{array}\right]$} & \multirow{3}{*}[25]{} & \multirow{3}{*}{ Predictor } \\
\hline$P_{m, l o d}, V_{x, r}$ & $P_{\text {loss }}$ & {$\left[\begin{array}{llll}5 & 15 & 10 & 5\end{array}\right]$} & & \\
\hline$P_{m, l o d}, V_{x, r}$ & $\dot{\chi}_{m e c h}$ & {$\left[\begin{array}{llll}5 & 15 & 10 & 5\end{array}\right]$} & & \\
\hline
\end{tabular}

Table A7. The left front tire 106(4) neural network configurations.

\begin{tabular}{|c|c|c|c|c|}
\hline Input Features & $\begin{array}{l}\text { Output } \\
\text { Features }\end{array}$ & $\begin{array}{l}\text { SANN Hidden } \\
\text { Layer Size }\end{array}$ & $\begin{array}{l}\text { LSTMN Hidden } \\
\text { Layer Size }\end{array}$ & Purpose \\
\hline $\begin{array}{l}P_{m, s r c}, P_{m, l o d} \\
\tau_{m, s r c}, \omega_{m, s r c}, \omega_{m, l o d} \\
P_{m, s r c}, P_{m, l o d}\end{array}$ & $\begin{array}{l}P_{\text {loss }} \\
P_{m, l o d} \\
\dot{\chi}_{\text {mech }}\end{array}$ & $\begin{array}{l}{\left[\begin{array}{ll}10 & 5\end{array}\right]} \\
{\left[\begin{array}{ll}10 & 5\end{array}\right]} \\
{\left[\begin{array}{ll}10 & 5\end{array}\right]}\end{array}$ & {$[25]$} & Estimator \\
\hline $\begin{array}{l}P_{m, l o d}, V_{x, r} \\
P_{m, l o d}, V_{x, r} \\
P_{m, l o d}, V_{x, r}\end{array}$ & $\begin{array}{l}P_{m, \text { src }} \\
P_{\text {loss }} \\
\dot{\chi}_{\text {mech }}\end{array}$ & $\begin{array}{l}{\left[\begin{array}{llll}5 & 15 & 10 & 5\end{array}\right]} \\
{\left[\begin{array}{llll}5 & 15 & 10 & 5\end{array}\right]} \\
{\left[\begin{array}{llll}5 & 15 & 10 & 5\end{array}\right]}\end{array}$ & {$[25]$} & Predictor \\
\hline
\end{tabular}


Table A8. The boost converter 205(1) neural network configurations.

\begin{tabular}{|c|c|c|c|c|}
\hline Input Features & $\begin{array}{l}\text { Output } \\
\text { Features }\end{array}$ & $\begin{array}{l}\text { SANN Hidden } \\
\text { Layer Size }\end{array}$ & $\begin{array}{l}\text { LSTMN Hidden } \\
\text { Layer Size }\end{array}$ & Purpose \\
\hline $\begin{array}{l}P_{e, s r c}, V_{e, s r c}, I_{e, s r c}, P_{e, l o d}, I_{e, l o d} \\
T_{t h}, T_{t h}-T_{t h, e x t} \\
P_{e, s r c}, V_{e, s r c}, I_{e, s r c}, P_{e, l o d}, I_{e, l o d} \\
T_{t h}, T_{t h, e x t}, T_{t h, a m b}, T_{t h}-T_{t h, e x t}\end{array}$ & $\begin{array}{l}P_{\text {loss }} \\
P_{\text {th,tran }} \\
\dot{\chi}_{\text {elec }} \\
\dot{\chi}_{\text {th }}\end{array}$ & $\begin{array}{l}{\left[\begin{array}{llll}10 & 25 & 25 & 15\end{array}\right]} \\
{\left[\begin{array}{lll}10 & 5\end{array}\right]} \\
{\left[\begin{array}{llll}10 & 25 & 25 & 15\end{array}\right]} \\
{\left[\begin{array}{llll}5 & 10 & 10 & 10\end{array}\right]}\end{array}$ & {$\left[\begin{array}{llll}10 & 25 & 25 & 5\end{array}\right]$} & Estimator \\
\hline $\begin{array}{l}P_{e, l o d}, V_{x, r}, I_{m i s, r} \\
P_{e, s r c}, P_{e, l o d}, V_{x, r}, I_{m i s, r} \\
T_{t h}, T_{t h}-T_{t h, e x t} \\
P_{e, s r c}, P_{e, l o d}, V_{x, r}, I_{m i s, r} \\
T_{t h}, T_{t h, e x t}, T_{t h, a m b}, T_{t h}-T_{t h, e x t}\end{array}$ & $\begin{array}{l}P_{e, s r c} \\
P_{\text {loss }} \\
P_{\text {th,tran }} \\
\dot{\chi}_{\text {elec }} \\
\dot{\chi}_{t h}\end{array}$ & 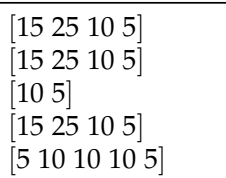 & {$\left[\begin{array}{llll}10 & 25 & 25 & 5\end{array}\right]$} & Predictor \\
\hline
\end{tabular}

Table A9. The buck converter 205(2) neural network configurations.

\begin{tabular}{|c|c|c|c|c|}
\hline Input Features & $\begin{array}{l}\text { Output } \\
\text { Features }\end{array}$ & $\begin{array}{l}\text { SANN Hidden } \\
\text { Layer Size }\end{array}$ & $\begin{array}{l}\text { LSTMN Hidden } \\
\text { Layer Size }\end{array}$ & Purpose \\
\hline $\begin{array}{l}P_{e, s r c}, V_{e, s r c}, I_{e, s r c}, P_{e, l o d}, I_{e, l o d} \\
T_{t h}, T_{t h}-T_{t h, e x t} \\
P_{e, s r c}, V_{e, s r c}, I_{e, s r c}, P_{e, l o d}, I_{e, l o d} \\
T_{t h}, T_{t h, e x t}, T_{t h, a m b}, T_{t h}-T_{t h, e x t}\end{array}$ & $\begin{array}{l}P_{\text {loss }} \\
P_{\text {th,tran }} \\
\dot{\chi}_{\text {elec }} \\
\dot{\chi}_{\text {th }}\end{array}$ & $\begin{array}{l}{\left[\begin{array}{llll}10 & 5 & 10 & 5\end{array}\right]} \\
{\left[\begin{array}{lll}10 & 5\end{array}\right]} \\
{\left[\begin{array}{lll}10 & 5 & 10\end{array}\right]} \\
{\left[\begin{array}{lll}5 & 5 & 5\end{array}\right]}\end{array}$ & {$\left[\begin{array}{llll}10 & 25 & 25 & 5\end{array}\right]$} & Estimator \\
\hline $\begin{array}{l}P_{e, l o d}, I_{\text {mis }, r} \\
P_{e, s r c}, P_{e, l o d}, V_{x, r} \\
T_{t h}, T_{t h}-T_{t h, e x t} \\
P_{e, s r c}, P_{e, l o d}, V_{x, r}, I_{m i s, r} \\
T_{t h}, T_{t h, e x t}, T_{t h, a m b}, T_{t h}-T_{\text {th,ext }}\end{array}$ & $\begin{array}{l}P_{e, s r c} \\
P_{\text {loss }} \\
P_{\text {th,tran }} \\
\dot{\chi}_{\text {elec }} \\
\dot{\chi}_{\text {th }}\end{array}$ & 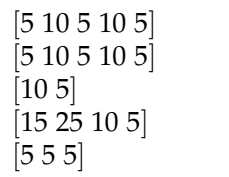 & {$\left[\begin{array}{llll}10 & 25 & 25 & 5\end{array}\right]$} & Predictor \\
\hline
\end{tabular}

Table A10. The buck converter 205(3) neural network configurations.

\begin{tabular}{|c|c|c|c|c|}
\hline Input Features & $\begin{array}{l}\text { Output } \\
\text { Features }\end{array}$ & $\begin{array}{l}\text { SANN Hidden } \\
\text { Layer Size }\end{array}$ & $\begin{array}{l}\text { LSTMN Hidden } \\
\text { Layer Size }\end{array}$ & Purpose \\
\hline $\begin{array}{l}P_{e, s r c}, V_{e, s r c}, I_{e, s r c}, P_{e, l o d}, I_{e, l o d} \\
T_{t h}, T_{t h}-T_{t h, e x t} \\
P_{e, s r c}, V_{e, s r c}, I_{e, s r c}, P_{e, l o d}, I_{e, l o d} \\
T_{t h}, T_{t h, e x t}, T_{t h, a m b}, T_{t h}-T_{t h, e x t}\end{array}$ & $\begin{array}{l}P_{\text {loss }} \\
P_{\text {th,tran }} \\
\dot{\chi}_{\text {elec }} \\
\dot{\chi}_{\text {th }}\end{array}$ & 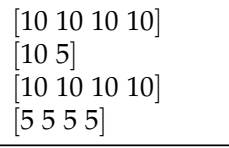 & {$\left[\begin{array}{llll}10 & 25 & 25 & 5\end{array}\right]$} & Estimator \\
\hline $\begin{array}{l}P_{e, l o d}, S S_{m, r}, \omega_{m, r} \\
P_{e, s r c}, P_{e, l o d}, S S_{m, r}, \omega_{m, r} \\
T_{t h}, T_{t h}-T_{t h, e x t} \\
P_{e, s r c}, P_{e, l o d}, S S_{m, r}, \omega_{m, r} \\
T_{t h}, T_{t h, e x t}, T_{t h, a m b}, T_{t h}-T_{t h, e x t}\end{array}$ & $\begin{array}{l}P_{e, s r c} \\
P_{\text {loss }} \\
P_{\text {th,tran }} \\
\dot{\chi}_{\text {elec }} \\
\dot{\chi}_{\text {th }}\end{array}$ & 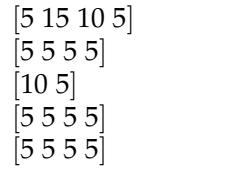 & {$\left[\begin{array}{llll}10 & 25 & 25 & 5\end{array}\right]$} & Predictor \\
\hline
\end{tabular}

Table A11. The cold plate 305(1) neural network configurations.

\begin{tabular}{|c|c|c|c|c|}
\hline Input Features & Output Features & SANN Hidden Layer Size & LSTMN Hidden Layer Size & Purpose \\
\hline $\begin{array}{l}\Psi_{t f, s r c}, T_{t f, s r c}, \dot{m}_{t f, s r c} \\
\Psi_{t f, s r c}, T_{t f, s r c}, \dot{m}_{t f, s r c} \\
\Psi_{t f, s r c}, T_{t f, s r c}, \dot{m}_{t f, s r c} \\
\dot{m}_{t f, s r c} \\
\dot{m}_{t f, s r c} \\
T_{t h, b a t t}, T_{t h, b a t t}-T_{t h, c p} \\
T_{t h, c p}, T_{t h, c p}-T_{t f, s r c}, \dot{m}_{t f, s r c}\end{array}$ & $\begin{array}{l}\Psi_{t f, l o d} \\
T_{t f, l o d} \\
\dot{m}_{t f, \text { lod }} \\
P_{t h, \text { tran }} \\
P_{t h, \text { rej }} \\
P_{t h, \text { cond }} \\
P_{\text {th,conv }}\end{array}$ & $\begin{array}{l}{\left[\begin{array}{llll}5 & 15 & 10 & 5\end{array}\right]} \\
{\left[\begin{array}{llll}5 & 15 & 10 & 5\end{array}\right]} \\
{\left[\begin{array}{llll}5 & 15 & 10 & 5\end{array}\right]} \\
{\left[\begin{array}{lll}5 & 10 & 5\end{array}\right]} \\
{\left[\begin{array}{lll}5 & 10 & 5\end{array}\right]} \\
{\left[\begin{array}{lll}10 & 5\end{array}\right]} \\
{\left[\begin{array}{llll}5 & 15 & 10 & 5\end{array}\right]}\end{array}$ & {$\left[\begin{array}{llll}10 & 25 & 25 & 5\end{array}\right]$} & Estimator \\
\hline $\begin{array}{l}\omega_{m, r} \\
\omega_{m, r}, \Xi, \tau_{o n}, \tau_{o f f} \\
T_{t h, c p}, T_{t h, c p}-T_{t f, l o d}, \omega_{m, r}, \Xi, \tau_{o n}, \tau_{o f f} \\
\Psi_{t f, s r c}, \omega_{m, r}, \Xi, \tau_{o n}, \tau_{o f f} \\
T_{t f, s r c}, \omega_{m, r}, \Xi, \tau_{o n}, \tau_{o f f} \\
\omega_{m, r} \\
\omega_{m, r}\end{array}$ & $\begin{array}{l}\dot{m}_{t f, \text { lod }} \\
\phi_{t f, s r c}-\phi_{t f, l o d} \\
P_{t h, \text { conv }} \\
\Psi_{t f, s r c}-\Psi_{t f, l o d} \\
T_{t f, \text { src }}-T_{t f, l o d} \\
P_{t h, \text { tran }} \\
P_{t h, \text { rej }}\end{array}$ & 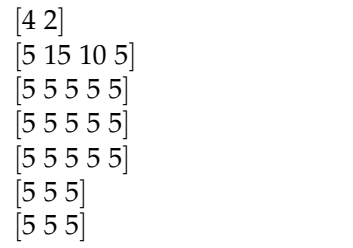 & {$\left[\begin{array}{llll}10 & 25 & 25 & 5\end{array}\right]$} & Predictor \\
\hline
\end{tabular}


Table A12. The coolant pump 315(1) neural network configurations.

\begin{tabular}{|c|c|c|c|c|}
\hline Input Features & Output Features & $\begin{array}{l}\text { SANN Hidden } \\
\text { Layer Size }\end{array}$ & $\begin{array}{l}\text { LSTMN Hidden } \\
\text { Layer Size }\end{array}$ & Purpose \\
\hline $\begin{array}{l}\Psi_{t f, s r c}, T_{t f, s r c}, \dot{m}_{t f, s r c} \\
\Psi_{t f, s r c}, T_{t f, s r c}, \dot{m}_{t f, s r c} \\
\Psi_{t f, s r c}, T_{t f, s r c}, \dot{m}_{t f, s r c} \\
\omega_{m, s r c} \\
\omega_{m, s r c} \\
\omega_{m, s r c} \\
T_{t h, b a t t}, T_{t h, b a t t}-T_{t h, c p} \\
T_{t h, c p}, T_{t h, c p}-T_{t f, s r c}, \dot{m}_{t f, s r c}\end{array}$ & $\begin{array}{l}\Psi_{t f, l o d} \\
T_{t f, \text { lod }} \\
\dot{m}_{t f, l o d} \\
P_{m, \text { src }} \\
P_{t h, t r a n} \\
P_{t h, \text { rej }} \\
P_{t h, \text { ond }} \\
P_{t h, \text { conv }} \\
\end{array}$ & 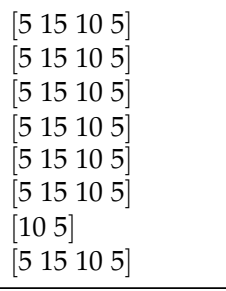 & {$\left[\begin{array}{llll}10 & 25 & 25 & 5\end{array}\right]$} & Estimator \\
\hline $\begin{array}{l}\omega_{m, r} \\
\omega_{m, r}, \Xi, \tau_{o n}, \tau_{o f f} \\
\omega_{m, r} \\
\Psi_{t f, s r c}, \omega_{m, r}, \Xi, \tau_{o n}, \tau_{o f f} \\
T_{t f, s r c}, \omega_{m, r}, \Xi, \tau_{o n}, \tau_{o f f} \\
\omega_{m, r} \\
\omega_{m, r}\end{array}$ & $\begin{array}{l}P_{m, s r c} \\
\phi_{t f, s r c}-\phi_{t f, l o d} \\
\dot{m}_{t f, l o d} \\
\Psi_{t f, s r c}-\Psi_{t f, l o d} \\
T_{t f, s r c}-T_{t f, l o d} \\
P_{t h, t r a n} \\
P_{t h, r e j}\end{array}$ & 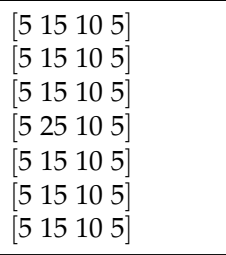 & {$\left[\begin{array}{llll}10 & 25 & 25 & 5\end{array}\right]$} & Predictor \\
\hline
\end{tabular}

Table A13. The coolant pump 321(1) neural network configurations.

\begin{tabular}{|c|c|c|c|c|}
\hline Input Features & $\begin{array}{l}\text { Output } \\
\text { Features }\end{array}$ & $\begin{array}{l}\text { SANN Hidden } \\
\text { Layer Size }\end{array}$ & $\begin{array}{l}\text { LSTMN Hidden Layer } \\
\text { Size }\end{array}$ & Purpose \\
\hline $\begin{array}{l}T_{t h}, T_{t h}-T_{t h, a m b} \\
T_{t h}, T_{t h, e x t}, T_{t h, a m b}, T_{t h}-T_{t h, e x t}\end{array}$ & $\begin{array}{l}P_{t h, r e j} \\
\dot{\chi}_{t h}\end{array}$ & $\begin{array}{l}{\left[\begin{array}{ll}10 & 5\end{array}\right]} \\
{\left[\begin{array}{llll}5 & 15 & 10 & 5\end{array}\right]}\end{array}$ & {$\left[\begin{array}{llll}10 & 25 & 25 & 5\end{array}\right]$} & Estimator \\
\hline $\begin{array}{l}T_{t h}, T_{t h}-T_{t h, a m b} \\
T_{t h}, T_{t h, e x t}, T_{t h, a m b}, T_{t h}-T_{t h, e x t}\end{array}$ & $\begin{array}{l}P_{t h, r e j} \\
\dot{\chi}_{t h}\end{array}$ & $\begin{array}{l}{\left[\begin{array}{ll}10 & 5\end{array}\right]} \\
{\left[\begin{array}{llll}5 & 15 & 10 & 5\end{array}\right]}\end{array}$ & {$\left[\begin{array}{llll}10 & 25 & 25 & 5\end{array}\right]$} & Predictor \\
\hline
\end{tabular}

Table A14. The coolant tank 322(1) neural network configurations.

\begin{tabular}{|c|c|c|c|c|}
\hline Input Features & Output Features & $\begin{array}{l}\text { SANN Hidden } \\
\text { Layer Size }\end{array}$ & $\begin{array}{l}\text { LSTMN Hidden } \\
\text { Layer Size }\end{array}$ & Purpose \\
\hline $\begin{array}{ll}\Psi_{t f, s r c} & T_{t f, s r c}, \dot{m}_{t f, s r c} \\
\Psi_{t f, s r c} & T_{t f, s r c}, \dot{m}_{t f, s r c} \\
\Psi_{t f, s r c} & T_{t f, s r c}, \dot{m}_{t f, s r c} \\
T_{t f, l o d} & T_{t f, l o d}-T_{t h, e x t} \\
T_{t f, l o d} & T_{t f, l o d}-T_{t h, e x t} \\
T_{t f, l o d}, & T_{t h, e x t}, T_{t h, a m b}, T_{t f, l o d}-T_{t h, e x t}\end{array}$ & $\begin{array}{l}\Psi_{t f, l o d} \\
T_{t f, l o d} \\
\dot{m}_{t f, l o d} \\
P_{t h, \text { tran }} \\
P_{t h, \text { rej }} \\
\dot{\chi}_{t h}\end{array}$ & 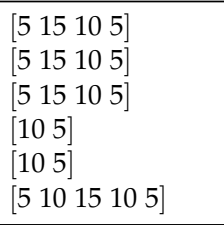 & {$\left[\begin{array}{llll}10 & 25 & 25 & 5\end{array}\right]$} & Estimator \\
\hline $\begin{array}{l}\omega_{m, r} \\
\omega_{m, r} \\
\omega_{m, r} \\
\omega_{m, r}, \Xi, \tau_{o n}, \tau_{o f f} \\
T_{t f, s r c}, \omega_{m, r}, \Xi, \tau_{o n}, \tau_{o f f} \\
T_{t f, l o d}, \omega_{m, r}, \Xi, \tau_{o n}, \tau_{o f f} \\
T_{t f, l o d}, T_{t f, l o d}-T_{t h, e x t} \\
T_{t f, l o d}, T_{t f, l o d}-T_{t h, e x t} \\
T_{t f, l o d}, T_{t h, e x t}, T_{t h, a m b}, T_{t f, l o d}-T_{t h, e x t}\end{array}$ & $\begin{array}{l}P_{m, s r c} \\
\phi_{t f, s r c}-\phi_{t f, l o d} \\
\dot{m}_{t f, l o d} \\
\Psi_{t f, s r c}-\Psi_{t f, l o d} \\
T_{t f, s r c}-T_{t f, l o d} \\
P s i_{t f, l o d} \\
P_{t h, t r a n} \\
P_{t h, r e j} \\
\dot{\chi}_{t h}\end{array}$ & 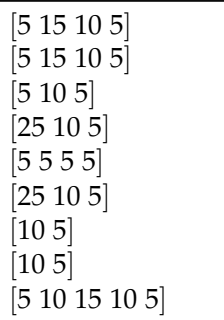 & {$\left[\begin{array}{llll}10 & 25 & 25 & 5\end{array}\right]$} & Predictor \\
\hline
\end{tabular}

Table A15. The coolant tank 323(1) neural network configurations.

\begin{tabular}{|c|c|c|c|c|}
\hline Input Features & Output Features & $\begin{array}{l}\text { SANN Hidden } \\
\text { Layer Size }\end{array}$ & $\begin{array}{l}\text { LSTMN Hidden } \\
\text { Layer Size }\end{array}$ & Purpose \\
\hline$\Psi_{t f, s r c}, T_{t f, s r c}, \dot{m}_{t f, s r c}$ & $\Psi_{t f, l o d}$ & {$\left[\begin{array}{ll}10 & 5\end{array}\right]$} & & \\
\hline$\Psi_{t f, s r c}, T_{t f, s r c}, \dot{m}_{t f, s r c}$ & $T_{t f, l o d}$ & {$\left[\begin{array}{llll}5 & 5 & 5 & 5\end{array}\right]$} & & \\
\hline$\Psi_{t f, s r c}, T_{t f, s r c}, \dot{m}_{t f, s r c}$ & $\dot{m}_{t f, l o d}$ & {$\left[\begin{array}{llll}5 & 15 & 10 & 5\end{array}\right]$} & & \\
\hline$\dot{m}_{t f, s r c}$ & $P_{m, l o d}$ & {$\left[\begin{array}{ll}4 & 2\end{array}\right]$} & {$\left[\begin{array}{llll}10 & 25 & 25 & 5\end{array}\right]$} & Estimator \\
\hline$\dot{m}_{t f, s r c}$ & $P_{t h, \text { tran }}$ & {$\left[\begin{array}{lll}5 & 5 & 5\end{array}\right]$} & & \\
\hline$\dot{m}_{t f, s r c}$ & $P_{t h, r e j}$ & {$\left[\begin{array}{lll}5 & 5 & 5\end{array}\right]$} & & \\
\hline$T_{t h, e x t}, T_{t h, a m b}$ & $\dot{\chi}_{t h}$ & {$\left[\begin{array}{lllll}5 & 10 & 15 & 10 & 5\end{array}\right]$} & & \\
\hline
\end{tabular}


Table A15. Cont.

\begin{tabular}{|c|c|c|c|c|}
\hline Input Features & Output Features & $\begin{array}{l}\text { SANN Hidden } \\
\text { Layer Size }\end{array}$ & $\begin{array}{l}\text { LSTMN Hidden } \\
\text { Layer Size }\end{array}$ & Purpose \\
\hline$\omega_{m, r}$ & $P_{m, l o d}$ & {$\left[\begin{array}{lll}5 & 15 & 5\end{array}\right]$} & \multirow{9}{*}[\begin{array}{llll}{10}&{25}&{25}&{5}\end{array}]{} & \multirow{9}{*}{ Predictor } \\
\hline$\omega_{m, r}$ & $\phi_{t f, s r c}-\phi_{t f, l o d}$ & {$\left[\begin{array}{llll}5 & 15 & 10 & 5\end{array}\right]$} & & \\
\hline$\omega_{m, r}$ & $\dot{m}_{t f, l o d}$ & {$\left[\begin{array}{llll}5 & 15 & 10 & 5\end{array}\right]$} & & \\
\hline$P s i_{t f, s r c}, \Xi, \tau_{o n}, \tau_{o f f}$ & $\Psi_{t f, s r c}-\Psi_{t f, l o d}$ & {$\left[\begin{array}{lll}25 & 10 & 5\end{array}\right]$} & & \\
\hline$T_{t f, s r c}, \omega_{m, r}, \Xi, \tau_{o n}, \tau_{o f f}$ & $T_{t f, s r c}-T_{t f, l o d}$ & {$\left[\begin{array}{llll}5 & 5 & 5 & 5\end{array}\right]$} & & \\
\hline$T_{t f, l o d}, \omega_{m, r}, \Xi, \tau_{o n}, \tau_{o f f}$ & $P s i_{t f, l o d}$ & {$\left[\begin{array}{lll}25 & 10 & 5\end{array}\right]$} & & \\
\hline$\omega_{m, r}$ & $P_{t h, t r a n}$ & {$\left[\begin{array}{lll}5 & 5 & 5\end{array}\right]$} & & \\
\hline$\omega_{m, r}$ & $P_{t h, r e j}$ & {$\left[\begin{array}{lll}5 & 5 & 5\end{array}\right]$} & & \\
\hline$T_{t h, e x t}, T_{t h, a m b}$ & $\dot{\chi}_{t h}$ & {$\left[\begin{array}{lllll}5 & 10 & 15 & 10 & 5\end{array}\right]$} & & \\
\hline
\end{tabular}

Table A16. The buck converter 400(1) neural network configurations.

\begin{tabular}{|c|c|c|c|c|}
\hline Input Features & $\begin{array}{l}\text { Output } \\
\text { Features }\end{array}$ & $\begin{array}{l}\text { SANN Hidden } \\
\text { Layer Size }\end{array}$ & $\begin{array}{l}\text { LSTMN Hidden } \\
\text { Layer Size }\end{array}$ & Purpose \\
\hline $\begin{array}{l}P_{e, s r c}, P_{m, l o d} \\
T_{t h}, T_{t h}-T_{\text {th,ext }} \\
P_{e, s r c}, P_{m, l o d} \\
P_{e, s r c}, P_{m, l o d} \\
T_{t h}, T_{t h, e x t}, T_{t h, a m b}, T_{t h}-T_{t h, e x t}\end{array}$ & $\begin{array}{l}P_{\text {loss }} \\
P_{\text {th,tran }} \\
\dot{\chi}_{\text {mech }} \\
\dot{\chi}_{\text {elec }} \\
\dot{\chi}_{\text {th }} \\
\end{array}$ & 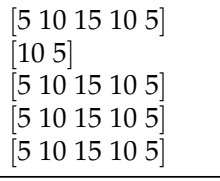 & {$\left[\begin{array}{llll}10 & 25 & 25 & 5\end{array}\right]$} & Estimator \\
\hline $\begin{array}{l}P_{m, l o d}, V_{x, r} \\
P_{e, s r c}, P_{m, l o d}, V_{x, r} \\
T_{t h}, T_{t h}-T_{t h, e x t} \\
P_{e, s r c}, P_{m, l o d}, V_{x, r} \\
P_{e, s r c}, P_{m, l o d}, V_{x, r} \\
T_{t h}, T_{t h, e x t}, T_{t h, a m b}, T_{t h}-T_{\text {th,ext }}\end{array}$ & $\begin{array}{l}P_{e, s r c} \\
P_{\text {loss }} \\
P_{\text {th,tran }} \\
\dot{\chi}_{\text {mech }} \\
\dot{\chi}_{\text {elec }} \\
\dot{\chi}_{\text {th }}\end{array}$ & 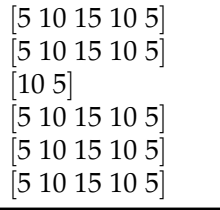 & {$\left[\begin{array}{llll}10 & 25 & 25 & 5\end{array}\right]$} & Estimator \\
\hline
\end{tabular}

Table A17. The buck converter 400(2) neural network configurations.

\begin{tabular}{|c|c|c|c|c|}
\hline Input Features & $\begin{array}{l}\text { Output } \\
\text { Features }\end{array}$ & $\begin{array}{l}\text { SANN Hidden } \\
\text { Layer Size }\end{array}$ & $\begin{array}{l}\text { LSTMN Hidden } \\
\text { Layer Size }\end{array}$ & Purpose \\
\hline $\begin{array}{l}P_{e, s r c}, P_{m, l o d}, \omega_{m, l o d} \\
T_{t h}, T_{t h}-T_{t h, e x t} \\
P_{e, s r c}, P_{m, l o d}, \omega_{m, l o d} \\
P_{e, s r c}, P_{m, l o d}, \omega_{m, l o d} \\
T_{t h}, T_{t h, e x t}, T_{t h, a m b}, T_{t h}-T_{t h, e x t}\end{array}$ & $\begin{array}{l}P_{\text {loss }} \\
P_{\text {th,tran }} \\
\dot{\chi}_{\text {mech }} \\
\dot{\chi}_{\text {elec }} \\
\dot{\chi}_{\text {th }} \\
\end{array}$ & $\begin{array}{l}{\left[\begin{array}{lllll}5 & 10 & 15 & 10 & 5\end{array}\right]} \\
{\left[\begin{array}{llll}10 & 5\end{array}\right]} \\
{\left[\begin{array}{lllll}5 & 10 & 15 & 10 & 5\end{array}\right]} \\
{\left[\begin{array}{lllll}5 & 10 & 15 & 10 & 5\end{array}\right]} \\
{\left[\begin{array}{lllll}5 & 10 & 15 & 10 & 5\end{array}\right]}\end{array}$ & {$\left[\begin{array}{llll}10 & 25 & 25 & 5\end{array}\right]$} & Estimator \\
\hline $\begin{array}{l}P_{m, l o d}, V_{x, r} \\
P_{e, s r c}, P_{m, l o d}, V_{x, r} \\
T_{t h}, T_{t h}-T_{t h, e x t} \\
P_{e, s r c}, P_{m, l o d}, \omega_{m, r} \\
P_{e, s r c}, P_{m, l o d}, \omega_{m, r} \\
T_{t h}, T_{t h, e x t}, T_{t h, a m b}, T_{t h}-T_{\text {th,ext }}\end{array}$ & $\begin{array}{l}P_{e, s r c} \\
P_{\text {loss }} \\
P_{\text {th,tran }} \\
\dot{\chi}_{\text {mech }} \\
\dot{\chi}_{\text {elec }} \\
\dot{\chi}_{\text {th }}\end{array}$ & 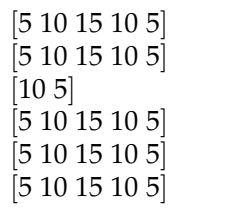 & {$\left[\begin{array}{llll}10 & 25 & 25 & 5\end{array}\right]$} & Estimator \\
\hline
\end{tabular}

Table A18. The battery's 502(1) neural network configurations.

\begin{tabular}{|c|c|c|c|c|}
\hline Input Features & $\begin{array}{l}\text { Output } \\
\text { Features }\end{array}$ & $\begin{array}{l}\text { SANN Hidden } \\
\text { Layer Size }\end{array}$ & $\begin{array}{l}\text { LSTMN Hidden } \\
\text { Layer Size }\end{array}$ & Purpose \\
\hline $\begin{array}{l}P_{\text {lod }}, V_{\text {lod }}, I_{\text {lod }} \\
P_{\text {src }}, P_{\text {lod }}, V_{\text {lod }}, I_{\text {lod }} \\
T_{\text {th }}, T_{\text {th }}-T_{\text {th,ext }} \\
T_{\text {th }}, T_{\text {th }}-T_{\text {th,cp }} \\
P_{\text {src }}, P_{\text {lod }}, V_{\text {lod }}, I_{\text {lod }} \\
T_{\text {th }}, T_{\text {th,ext }}, T_{\text {th,amb }}, T_{\text {th }}-T_{\text {th,ext }}, T_{\text {th }, c p}\end{array}$ & $\begin{array}{l}P_{\text {src }} \\
P_{\text {loss }} \\
P_{\text {th,tran }} \\
P_{\text {th,con }} \\
\dot{\chi}_{\text {elec }} \\
\dot{\chi}_{\text {th }}\end{array}$ & 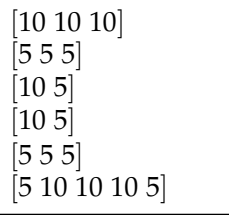 & {$\left[\begin{array}{llll}10 & 25 & 25 & 5\end{array}\right]$} & Estimator \\
\hline $\begin{array}{l}P_{\text {lod }}, V_{x, r}, I_{m i s, r} \\
P_{s r c}, P_{\text {lod }}, V_{x, r}, I_{m i s, r} \\
T_{t h}, T_{t h}-T_{t h, e x t} \\
T_{t h}, T_{t h}-T_{t h, c p} \\
P_{s r c}, P_{l o d}, V_{x, r}, I_{m i s, r} \\
T_{t h}, T_{t h, e x t}, T_{t h, a m b}, T_{t h}-T_{t h, e x t}, T_{t h, c p}\end{array}$ & $\begin{array}{l}P_{\text {src }} \\
P_{\text {loss }} \\
P_{\text {th,tran }} \\
P_{\text {th,con }} \\
\dot{\chi}_{\text {elec }} \\
\dot{\chi}_{\text {th }}\end{array}$ & 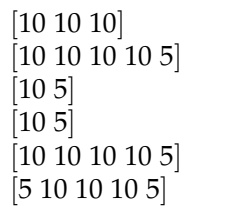 & {$\left[\begin{array}{llll}10 & 25 & 25 & 5\end{array}\right]$} & Predictor \\
\hline
\end{tabular}




\section{Appendix B}

The intent of this section is to provide an example of how the first law of thermodynamics (FLT) and second law of thermodynamics (SLT) was used to derive relevant exergy based principles.

\section{Appendix B.1}

This Appendix contains a derivation of the DC Motor/Generator FLT and SLT.

Step 1: We define our electrical-mechanical based components which approximate a DC spinning machine, and added a lumped parameter thermal model. The resulting Electrical-Mechanical-Thermal Circuit is provided in Figure A1, the equations are also provided in Jane et al. [26].

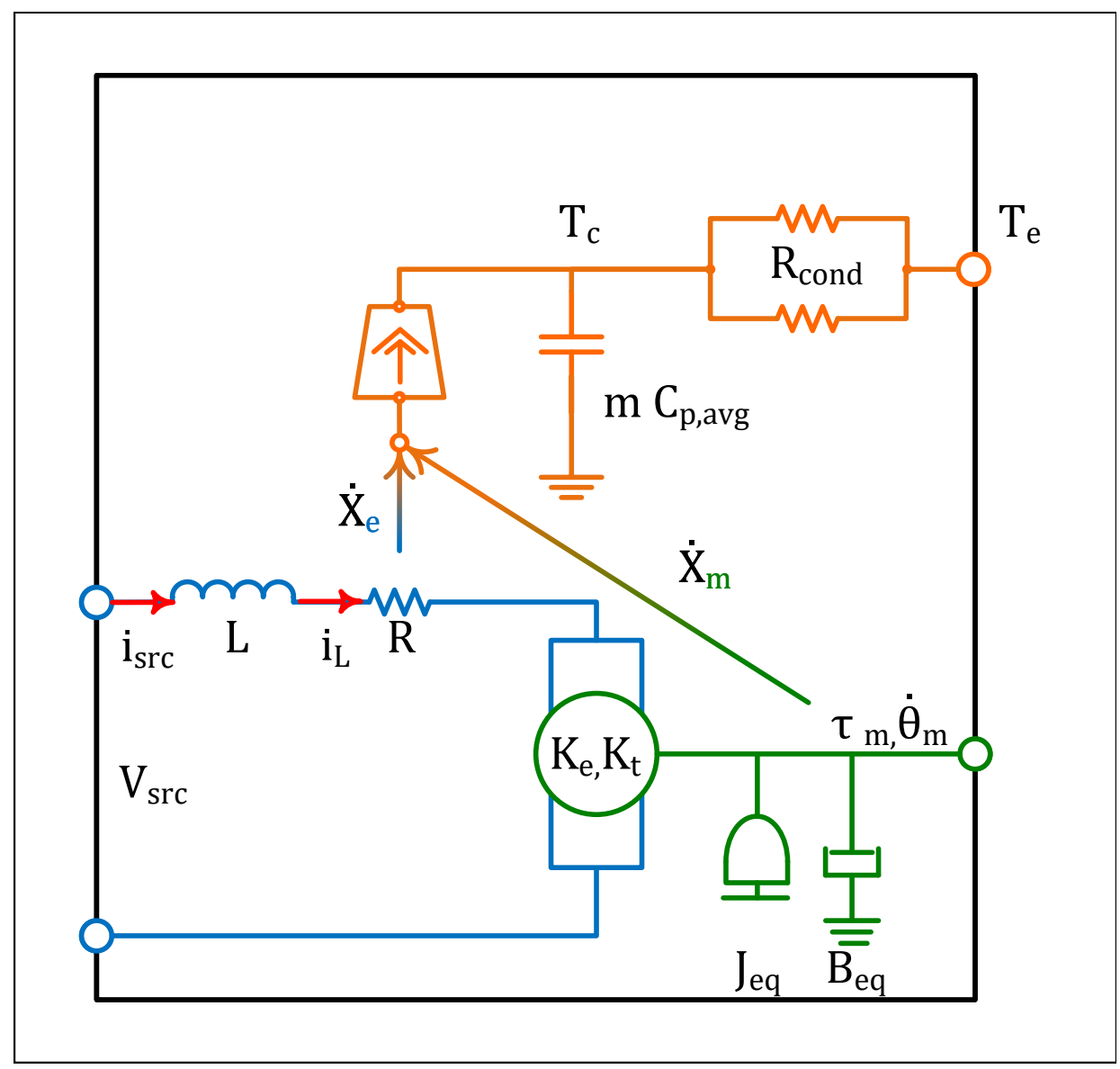

Figure A1. Electrical-Mechanical-Thermal Circuit Used for Model DC Motor/Generator.

Step 2: We derive the equations of motion which approximate a DC Motor/Generator electrical-mechanical system of equations:

$$
\begin{gathered}
L \frac{d i_{L}}{d t}=V_{s r c}-i_{l} R-K_{e} \omega_{m} \\
J_{e q} \frac{d^{2} \omega_{m}}{d t^{2}}=K_{t} i_{L}-B_{e q} \frac{d \omega_{m}}{d t-\tau_{l o d}}
\end{gathered}
$$

Step 3: An energy balance is preformed for the electrical-mechanical based system:

$$
P_{s r c}-P_{\text {lod }}=P_{\text {str }}+P_{\text {loss }}
$$

where

$$
P_{s r c}=V_{s r c} i_{L}
$$




$$
\begin{gathered}
P_{\text {lod }}=\tau_{\text {lod }} \omega_{m} \\
P_{\text {str }}=L \frac{d i_{L}}{d t} i_{L}+J_{e q} \frac{d^{2} \omega_{m}}{d t^{2} \omega_{m}} \\
P_{\text {loss }}=\dot{\chi}_{e m}=\overbrace{i_{L}^{2} R}^{\dot{x}_{e l e c}}+\overbrace{\omega_{m}^{2} B_{e q}}^{\dot{x}_{m e c h}}
\end{gathered}
$$

There are two loss terms, each of them represents a different exergetic losses for the DC Motor/Generator that must be overcome to produce sufficient energy to drive the corresponding load. In the case of the DC Motor/Generator $P_{\text {loss }}$ represents pure exergetic losses of the system, i.e., the $i_{L}^{2} R$ is the pure exergetic losses ( $\left.\dot{\chi}_{\text {elec }}\right)$ of the electrical circuit, where as $\omega_{m}^{2} B_{e q}$ is the pure mechanical exergetic loss $\left(\dot{\chi}_{m e c h}\right)$ of the mechanical based components of the DC Motor/Generator. The aggregate is assumed to be $\dot{\chi}_{e m}$. The energy dissipated by these components represents the unusable energy which we must overcome to obtain usable energy out of the system, i.e., exergy.

Step 4: The aggregate electrical and mechanical exergetic losses then fuel the lumped parameter thermal model of the system given by the FLT:

$$
\dot{\chi}_{e m}=m C_{p, a v g} \dot{T}_{c}+2 R_{\text {cond }}\left(T_{c}-T_{e}\right)
$$

where $\frac{d T_{c}}{d t}=\dot{T}_{c}$.

Step 5: Because there is a thermal circuit and therefor energy may transfer from the asset to anther asset, we must also perform an entropy balance (SLT):

$$
\frac{\dot{\chi}_{e m}}{T_{\mathcal{C}}}+\dot{S}_{\text {gen }}=\frac{m C_{p, a v g} \dot{T}_{c}}{T_{\mathcal{c}}}+\frac{2 R_{\text {cond }}\left(T_{\mathcal{c}}-T_{e}\right)}{T c}
$$

Substituting in the energy balance (combine FLT and SLT), resulting in:

$$
\dot{S}_{\text {gen }}=\frac{2 R_{\text {cond }}\left(T_{c}-T_{e}\right)^{2}}{T_{c} T_{e}}
$$

Step 6: Thus the thermal exergetic losses is then:

$$
\dot{\chi}_{t h}=T_{o} \dot{S}_{g e n}
$$

The $\dot{\chi}_{t h}$ exergetic losses is a direct result of heat being allowed to flow and the fact that there is a temperature difference. If $T_{c}=T_{e}=T_{o}$ then there is no additional exergetic losses for the system. Any thermal variation will subsequently lead to some type of destroyed exergy.

\section{References}

1. Force, U.A. ‘Tanks' for the Ride. Available online: https://www.af.mil/News/Article-Display/Article/112093/tanks-for-theride/ (accessed on 16 February 2021).

2. Li, F.; Peng, X.; Wang, Z.; Zhou, Y.; Wu, Y.; Jiang, M.; Xu, M. Machine Learning (ML)-Assisted Design and Fabrication for Solar Cells. Energy Environ. Mater. 2019, 2, 280-291. [CrossRef]

3. Serale, G.; Fiorentini, M.; Capozzoli, A.; Bernardini, D.; Bemporad, A. Model Predictive Control (MPC) for Enhancing Building and HVAC System Energy Efficiency: Problem Formulation, Applications and Opportunities. Energies 2018, 11, 631. [CrossRef]

4. Sharma, A.; Kushvaha, V. Predictive modelling of fracture behaviour in silica-filled polymer composite subjected to impact with varying loading rates using artificial neural network. Eng. Fract. Mech. 2020, 239, 107328. [CrossRef]

5. Kushvaha, V.; Kumar, S.A.; Madhushri, P.; Sharma, A. Artificial neural network technique to predict dynamic fracture of particulate composite. J. Compos. Mater. 2020, 54, 3099-3108. [CrossRef]

6. Hassoun, M.H. Fundamentals of Artificial Neural Networks; MIT Press: Cambridge, MA, USA, 1995.

7. Haykin, S.; Network, N. A comprehensive foundation. Neural Netw. 2004, 2, 41.

8. Goodfellow, I.; Bengio, Y.; Courville, A. Deep Learning; MIT Press: Cambridge, MA, USA, 2016. Available online: http: //www.deeplearningbook.org (accessed on 16 February 2021), 
9. Jung, H.; Choi, M.K.; Jung, J.; Lee, J.H.; Kwon, S.; Jung, W.Y. ResNet-based vehicle classification and localization in traffic surveillance systems. In Proceedings of the IEEE Conference on Computer Vision and Pattern Recognition Workshops, Honolulu, HI, USA, 21-26 July 2017; pp. 61-67. [CrossRef]

10. Simonyan, K.; Zisserman, A. Very deep convolutional networks for large-scale image recognition. arXiv 2014, arXiv:1409.1556 .

11. Krizhevsky, A.; Sutskever, I.; Hinton, G.E. Imagenet classification with deep convolutional neural networks. Adv. Neural Inf. Process. Syst. 2012, 25, 1097-1105. [CrossRef]

12. He, K.; Zhang, X.; Ren, S.; Sun, J. Deep residual learning for image recognition. In Proceedings of the IEEE conference on computer vision and pattern recognition, Las Vegas, NV, USA, 27-30 June 2016; pp. 770-778. [CrossRef]

13. Zhang, Z. ResNet-Based Model for Autonomous Vehicles Trajectory Prediction. In Proceedings of the 2021 IEEE International Conference on Consumer Electronics and Computer Engineering (ICCECE), Guangzhou, China, 15-17 January 2021; pp. 565-568. [CrossRef]

14. Yeon, K.; Min, K.; Shin, J.; Sunwoo, M.; Han, M. Ego-vehicle speed prediction using a long short-term memory based recurrent neural network. Int. J. Automot. Technol. 2019, 20, 713-722. [CrossRef]

15. Chen, J.; Zhao, P.; Liang, H.; Mei, T. Motion planning for autonomous vehicle based on radial basis function neural network in unstructured environment. Sensors 2014, 14, 17548-17566. [CrossRef] [PubMed]

16. Liu, W.; Shoji, Y. Applying Deep Recurrent Neural Network to Predict Vehicle Mobility. In Proceedings of the 2018 IEEE Vehicular Networking Conference (VNC), Taipei, Taiwan, 5-7 December 2018; pp. 1-6. [CrossRef]

17. Abadi, M.; Barham, P.; Chen, J.; Chen, Z.; Davis, A.; Dean, J.; Devin, M.; Ghemawat, S.; Irving, G.; Isard, M.; et al. Tensorflow: A system for large-scale machine learning. In Proceedings of the 12th \{USENIX\} symposium on operating systems design and implementation (OSDI 16), Savannah, GA, USA, 2-4 November 2016; pp. 265-283.

18. Chen, Z.; Yang, C.; Fang, S. A convolutional neural network-based driving cycle prediction method for plug-in hybrid electric vehicles with bus route. IEEE Access 2019, 8, 3255-3264. [CrossRef]

19. Yoon, S.; Kum, D. The multilayer perceptron approach to lateral motion prediction of surrounding vehicles for autonomous vehicles. In Proceedings of the 2016 IEEE Intelligent Vehicles Symposium (IV), Gotenburg, Sweden, 19-22 June 2016; pp. 1307-1312. [CrossRef]

20. McBride, S.; Sandu, C.; Alatorre, A.; Victorino, A. Estimation of Vehicle Tire-Road Contact Forces: A Comparison between Artificial Neural Network and Observed Theory Approaches; WCX World Congress Experience; SAE International: Detroit, MI, USA, 2018. [CrossRef]

21. Boada, B.L.; Boada, M.J.L.; Vargas-Melendez, L.; Diaz, V. A robust observer based on $H_{\infty}$ filtering with parameter uncertainties combined with Neural Networks for estimation of vehicle roll angle. Mech. Syst. Signal Process. 2018, 99, 611-623. [CrossRef]

22. Silberberg, M.; Duran, R. Chemistry: The Molecular Nature of Matter and Change; McGraw-Hill; New York, NY, USA, 2002.

23. Bennett, S. A brief history of automatic control. IEEE Control Syst. Mag. 1996, 16, 17-25. [CrossRef]

24. James, C.; Kim, T.Y.; Jane, R. A review of exergy based optimization and control. Processes 2020, 8, 364. [CrossRef]

25. Clearpath Robotics: Mobile Robots for Research \& Development. Available online: https://clearpathrobotics.com/ (accessed on 29 September 2019).

26. Jane, R.; Kim, T.; James, C.M. Developing Prediction Based Algorithms for Energy and Exergy Flow. Technical Report, SAE Technical Paper. 2021. Available online: https://www.sae.org/publications/technical-papers/content/2021-01-0258/ (accessed on 16 February 2021).

27. The Mathworks, Inc. Pipe (TL). Available online: https://www.mathworks.com/help/physmod/simscape/ref/pipetl.html (accessed on 16 February 2021).

28. The Mathworks, Inc. Fixed-Displacement Pump (TL). Available online: https://www.mathworks.com/help/physmod/hydro/ ref/fixeddisplacementpumptl.html?searchHighlight=Fixed-Displacement\%20Pump \%20\%28TL\%29\&s_tid=srchtitle (accessed on 16 February 2021).

29. The Mathworks, Inc. Tank (G-TL). Available online: https://www.mathworks.com/help/physmod/hydro/ref/tankgtl.html? searchHighlight=Tank $\% 20 \% 28 \mathrm{G}-\mathrm{TL} \% 29 \&$ s_tid=srchtitle (accessed on 16 February 2021).

30. Zhou, Q.; Chen, H.; Zhao, H.; Zhang, G.; Yong, J.; Shen, J. A Local Field Correlated and Monte Carlo Based Shallow Neural Network Model for Non-Linear Time Series Prediction; University of Wollongong Australia Online Research Database: Wollongong, Australia, 2016.

31. Aijun, L.; Hejun, L.; Kezhi, L.; Zhengbing, G. Applications of neural networks and genetic algorithms to CVI processes in carbon/carbon composites. Acta Mater. 2004, 52, 299-305. [CrossRef]

32. Fischer, T.; Krauss, C. Deep learning with long short-term memory networks for financial market predictions. Eur. J. Oper. Res. 2018, 270, 654-669. [CrossRef]

33. Woodbridge, J.; Anderson, H.S.; Ahuja, A.; Grant, D. Predicting domain generation algorithms with long short-term memory networks. arXiv 2016, arXiv:1611.00791 . 
34. Jane, R.S.; James, C.; Kim, T. Using AI-ML to develop energy and exergy flow characterizations for multi-domain operations In Artificial Intelligence and Machine Learning for Multi-Domain Operations Applications III; International Society for Optics and Photonics: 2021; Volume 11746, p. 1174628. Available online: https:/ / www.spiedigitallibrary.org/conference-proceedings-ofspie/11746/1174628/Using-AI-ML-to-develop-energy-and-exergy-flow-characterizations (accessed on 16 February 2021).

35. Sayin Kul, B.; Kahraman, A. Energy and Exergy Analyses of a Diesel Engine Fuelled with Biodiesel-Diesel Blends Containing 5 bioethanol. Entropy 2016, 18, 387. [CrossRef] 